\title{
f. Generalized Modal Shock Spectra Method for Spacecraft Loads Analysis
}

M. Trubert

M. Salama

National Aeronautics and Space Administration

Jet Propulsion Laboratory

California Institute of Technology

Pasadena, California 


\section{A Generalized Modal Shock Spectra Method for Spacecraft Loads Analysis}
M. Trubert
M. Salama

March 15, 1979

National Aeronautics and

Space Administration

Jet Propulsion Laboratory

Cá.ifornıa institute of Technology

Pasadena, Salifornia 
The research described in this publication was carried out by the Jet Propulsion Laboratory, California Institute of Technology, under NASA Contract No. NAST-100. 
The present work is a generalization of the original work, published and unpublished, pioneered by Robert H. Bamford of the Applied Mechanics Technology Section of JPL. Full acknowledgement is given to him for generating the fundamental ideas of making use of the shock spectra concept to determine bounds of loads in a spacecraft structure in the boost configuration. The effort was supported by the Office of Aeronautics and Space Technology. 
Unlike an earlier shock spectra approach, the generalization presented in this paper permits an accurate elastic interaction between the spacecraft and launch vehicle to obtain accurate bounds on the spacecraft response and structural loads. In addition, the modal response from a previcus launch vehicle transient analysis - with or without a dummy spacecraft - is explnited to define a modal impulse as a simple idealization of the actual forcing function. The idealized modal forcing function is then used to derive explicit expressions for an estimate of the bound on the spacecraft structural response and f'orces.

Greater accuracy is achieved with the present method over the earlier shock spectra, while saving much computational effort over the transient analysis. 



\section{CONTENTS (Continued)}

\section{APPENDIXES}

A.

ANALOG SOLUTION OF THE EQUATIONS OF MOTION

$A-1$

B. ANALYTICAL, SOLUTION OF THE EQUATIONS OF MOTION --..-- E-1

C. RECOVERY OF LAUNCH VEHICLE MODAL PROPERTIES -------- C-1

D. DISFLACEMENT SHECTRA FOR THE GENERALIZED RIGID BODY MODES

\section{Figures}

\begin{tabular}{|c|c|}
\hline $1-1$. & Shock Spectrum $S(\omega, \xi)$ of a Function $\ddot{x}_{0}(t)$ \\
\hline $1-2$. & Composite System of Spacecraft and Launch Vericle ---- \\
\hline $1-3$. & Idealization of Forcing Function \\
\hline $3-1$ & $\begin{array}{l}\text { Graphic Representation of } \mathrm{D}_{1}(t), \mathrm{D}_{2}(t) \text {, and } \\
\text { Their Approximate Maxima } \mathrm{D}_{1 \max } \text { and } \mathrm{D}_{2 \max }\end{array}$ \\
\hline $3-2$. & $\begin{array}{l}\text { Modal Displacement Spectra } D_{\ell} \text { and } Q \ell \text { for } a \\
\text { Launch Vehicle Mode } \ell\end{array}$ \\
\hline $3-3$ & Shock Spectra Matching and Envelope \\
\hline $4-1$ & Generalized Shock Spectra Method ---- \\
\hline$A-1$. & T,aunch Vehicle - Spacecraft Modal Model - \\
\hline$A-2$ & Analog Schematic for Initial Velocity Solution - \\
\hline A-3. & Analog Schematic for Impulse Solution -- \\
\hline$A-4$ & Mass Number 2 Response to Unit Impulse \\
\hline$A-5$ & Generalized Shock Spectra \\
\hline A-6. & Reduction Coefficient $\mathrm{C}$ \\
\hline $\mathrm{C}-1$. & $\begin{array}{l}\text { Composite Launch Vehicle With Elastic } \\
\text { Loaded Interface }\end{array}$ \\
\hline
\end{tabular}

\section{Table}

A -1 . 


\section{ORIGLNAL PACE
OF POOR QUALEY}

A. BACKGROUND

1. Transient Analysis

The determination of the internal loads in a spacecraft structure subjected to a dynamic launch environment is an iterative process aimed at the ultimate sizing of the structure for its design. The process is iterative because the internal loads in the spacecraft are dependent upon the spacecraft mass and stiffness distribution, as well as the mass and stiffness distribution interaction with the launch vehicle. Some of the key parameters that dominate the loads analysis process are timeliness of the sizing, cost, weight limitations, and low sensitivity to iterative changes in the design. Because the loads are time-dependent functions, a complete transient analysis aimed at determining responses to given time-dependent forcing functions is widely used throughout the industry to determine loads in the launch vehicle and in the spacecraft. The transient analysis is a simulation method by which the loads time histories must be computed in their entirety before peak values can be identified for establishing design loads.

Although a transient analysis tends to result in lightweight designs when properly used, it is usually costly and time consuminf, especially when several organizations are involved. No better method is presently known when dealing with a launch vehicle, and it is not sugfested here that this process be changed for the launch vehicles. An alternate method is presented, however, for the analysis of the spacecraft.

2. Alternates to Transient Analysis

In the anulysis of an aerospace structure, at least two organizations are involved: one organization responsible for the design and analysis of the launch vehicle, and another for the spacecraft. For the spacecraft-developing organization, it is desirable to perform frequent but inexpensive loads analyses to account for frequent desifn 
changes in the spacecraft. Ideally. these analyses would include only the spacecraft, without requiring launch vehicle modeling and without requiring activity at the launch vehicle agency. For this reason, several alternatives have been suggested, and References $1-?$ and $1-2$ are recent examples. Like the method presented here, the methods given in References 1-1 and 1-2 apply only when the loads in the spacecraft result trom time-dependent forcing functions applied on the launch vehicle, and bypass most of the costly time consuming steps of a complete transient solution.

\section{Reduced Transient Analysis Method}

The method of Reference $1-1$ is a reduced transient analysis. Accelerations at the spacecraft-launch vehicle interface are obtained by a single transient analysis of the launch vehicle with a rigid mass. From this single launch vehicle analysis, interface accelerations can then be corrected to account for the elasticity of the spacecraft and used as input to the spacecraft. The correction procedure requires know]edge of the characteristics of the elastic spacecraft and of the rigid mass/elastic launch vehicle, but does not require the actual integration of the spacecraft elastic model with the launch vehicle elastic model, resulting in a significant cost reduction. From there on, the procedure follows the usual transient analysis.

4. Shock Spectra Method

Like the approach presented here, the method of Reference 1-2 is a generalization of the trialiticnal shock spectra concept. In this concept, shown in Figure $1-1$, a shock spectrum $S(\omega, \xi)$ of a function $\ddot{x}_{0}(t)$ is the largest peak response of a single-degree-of-freedom oscillator subjected to the base forcing function, $\ddot{x}_{0}(t)$. The implicit assumption is made that the input forcing function, $\ddot{x}_{0}(t)$, is not affected by the presence of the oscillator. This is true only if the mass of the oscillator is infinitesimal.

The most significint feature of Reference $1-2$ was the introduction of the relative impedance between the spacecraft and the launch vehicle, 

i.e., between the oscillator and the base, in the form of a reduction factor, $C$, which accounts for the effect of the osciliator on the interface acceleration. In addition, an envelope is constructed for the acceleration spectrum at the interface between the spacecraft and launch vehicle, Figure 1-2, which is used to deterrine the level of an equivalent sinusoidal forcing function to be applied at the interface fur the computation of the spacecraft member 1uads. In this manner, spacecraft loads are computed with no integrated launch ve! icle/spacecraft transient analysis. This method was applied to several spacecraft structures as reported in Reference $1-3$, and was found to be much less costly to use, but produced somewhat heavier structures than a transient analysis.

In the application of the early shock spectra method outilined above, a number of assumptions were introduced that made its successful usage highly dependent upon the ane'yst's intuitive ability to recognize launch vehicle modes that are important to retain, and those of insignificant effects that can be discarded. The method sensitivity to the analyst's judgment is highlighted in the tacit assumption that the spacecraft/launch vehicle interface response is doninated alnust totally by one launch vehicle mode at any one frequency, and in the process by which the envelope of the shock spectra is constructed.

\section{The Generalized Shock Spectra Method}

The present approach is an extension of Reference 1-2, with the objective of eliminating the analyst's judgment, formalizing the derivation and computational steps of the approach, explicitly stating the necessary assumptions, relaxing unessential ones, and introducing several significant improvements. These will be discussed in subsequent sections.

B. OUTLINE OF THE GENERAI,IZED SHOCK SPECTRA

The following general observations are fundamental to understanding the rationale of the shock spectra approaches:

(1) The general objective is to avoid the cost of a launch vehicle/new spacecraft overall transient analysis. 


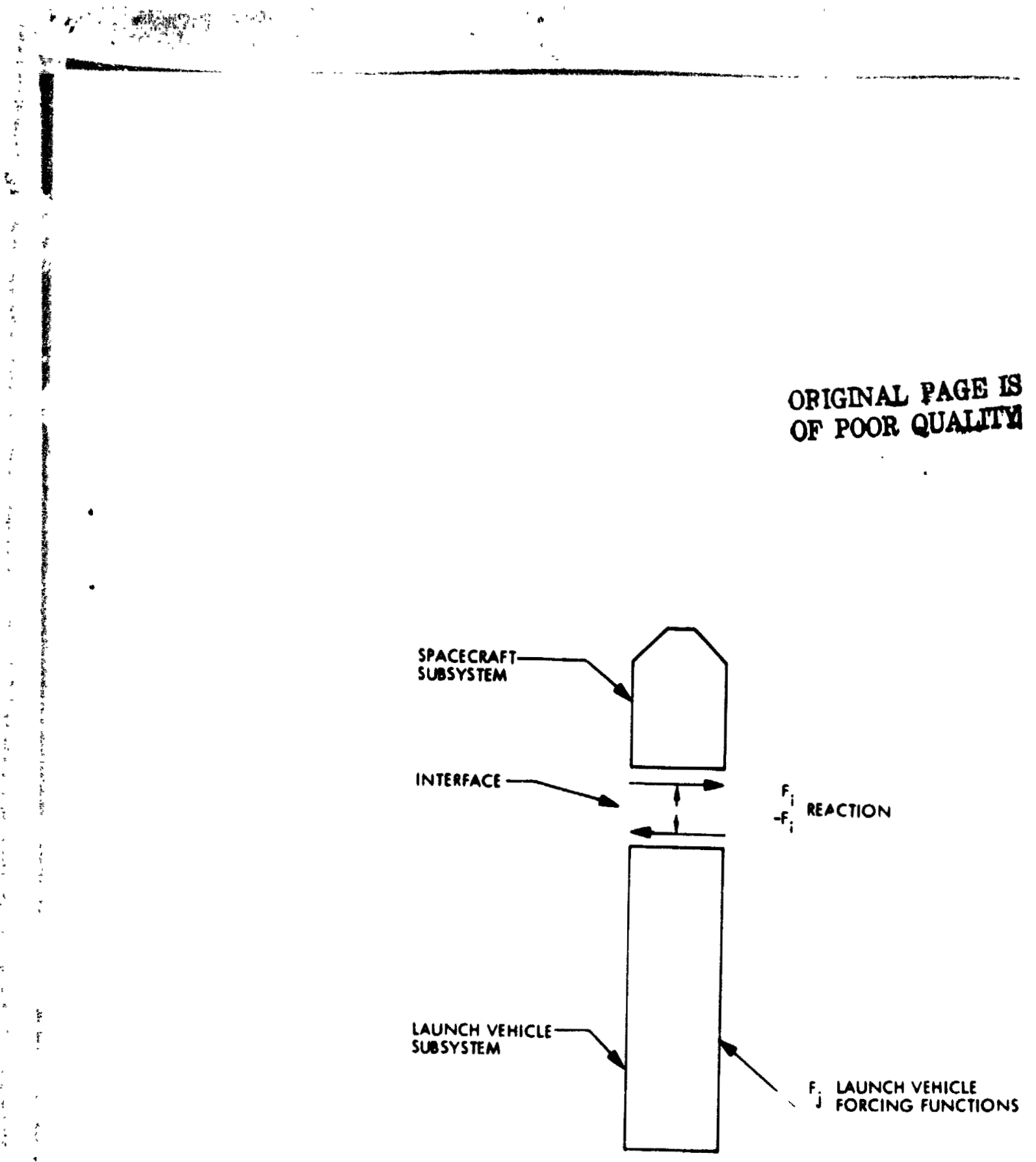

ORIGNAL PAGE IS
OF POOR QUAJIII

Figure 1-2. composite System of Spaceoratt and launch vehicle 
(2) There has been a previous dynamic analysis done for the launch vehicle with another or a dummy spacecraft, the results of which are used as inputs to the generalized shock spectra method. Note that the present method is derived for use of the modes of the launch vehicle loaded, by a rigid mass only, or not loaded. If zuch an analysis is not available, the proper data can still be $r$ covered from the analysis of the launch vehicle loaded with an elastic spacecraft as shown in $\dot{h}_{i}$ jendix $C$.

(3) The structural analyst needs only to determine the worst case load maxima or bounds, rather than time histories, of the structural response. Since maxima or bounds are the objective of the shock spectra concept, a shock spectra approach is readily applicable to the structural design prociess.

(4) Any loads analysis - transient, shock spectra, or other incorporates two basic items: a model idealizing the dynamic environment, i.e., the forcing function, and another idealizing the composite structural system. The form of each model is influenced by the selected approach, while its complexity is constrained by cost and time.

(5) The original launch vehicle forcing functions, usually unknown to the spacecraft analyst, may be idedized by a simpler form to obtain an explicit closed form solution. A complete definition of this idealization is possible from the previous analysis of item ( 1 ) above, and is done at the modal level.

In view of these remarks, the generalized shock spectra method is hiphliphted as follows:

First, the modal forcing function $F_{\ell}(t)$ corresponding to the modes of the launch vehicle loaded by a rigid mass at, the spacecraft interface, and representing the modal contribution of an actu flight 
event, is modeled, regardless of its physical puint of application, by an equivalent lounch vehicle modal forcing function, $F_{e l}(t)$. Unlike the actual complex transiert force, the equivalent furcing function chosen is a simpler form of variatiun with time. Here, an impulse delta function $F_{e \ell}(t)=F_{0 \ell} \delta(\tau)$ with a yet-lunknown magnitude $F_{0 \ell}$, or equivalently a velocity with magiltude $v_{0 \ell}$, is chosen for convenience.

The choice of a simplified forcing function as an impulse emphasizes the view that the shape of the response time history is of no consequence, and that only the peak or bound uf the response is of interest. Therefore, any forcing function that would reproduce a resporise with the same naximum peak or bound as the actual forcinf, function is acceptable. The equivalency between the actual forcing function and the idealized one is established, not un the basis of producing identical response time histories, but on the basis of producing an identical peak of the shock spectra of each of the launch velicle modal response $\ddot{q}_{l}(t)$, Figure $1-3$, derived from the previously performed launch vehicle/dummy spacecraft analysis. An alternate to the direct use of $\ddot{q}_{\ell}(t)$ is discussed later.

The use of modal shock spectra, rather than the interface degreesof-rreedom shock spectra is significant bec uuse it automaticaliy accounts for the matrhing of all interface physical degrees of freedom, and allows one to determine the modal magnitude of the impulse $F_{0 l}$ or velocity $v_{0 \ell}$. It is noteworthy that the above process of establishing the equivalent idealized forcing function requires knowledge of the modal properties of the launch vehicle with or without a payload. Such information is usually available from the launch vehicle organization. Also note that the rigid mass at the launch vehicle/spacecraft interface does not have to be the total mass of the spacecraft to be analyzed, but can have any value convenient for the transient analysis performed earlier on the launch vehicle. The present approach does correct for whatever mass value was used.

Second, in considering the composite structural system of Figure 1-2, which consists of a spacecraft modeled by S-normal modes and a launch vehicle modeled by L-normal modes, there will be $(S+L)$ 

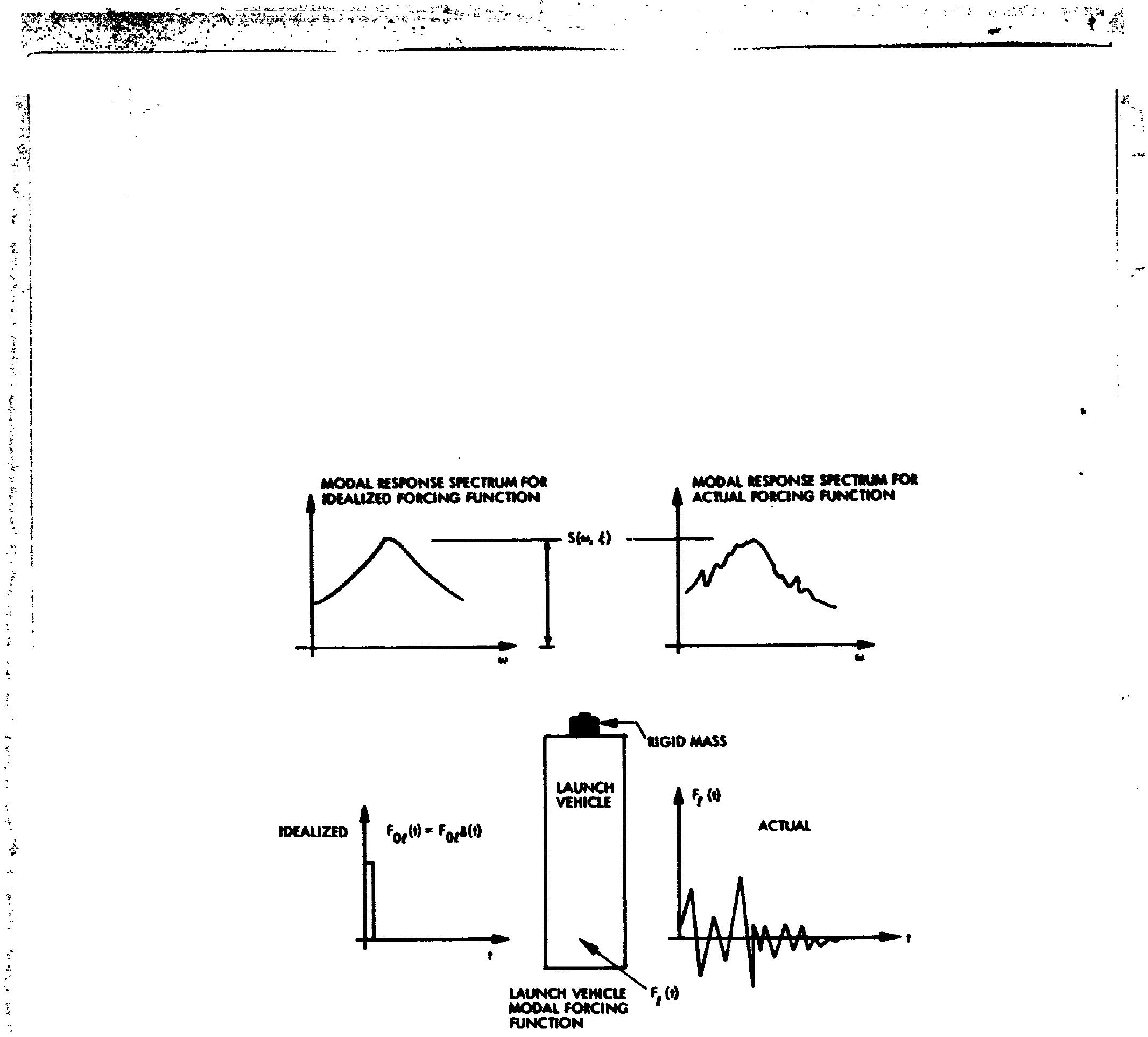

Figure 1-3. Idealization of Forcing Function 
modally coupled equations of motion. Unlike the transfent analysis where the solution is expressed in the complete $(S+L)$ space of modal coordinates and time, a bound on the complete solution is established herein by:

(1) Idealizing the totality of $(S+L)$ mathematical space of modal coordinates by an array of nested $(S \times L)$ mathematical subspaces, in each of which only one spacecraft mode is individualiy coupled with one launch vehicle mode. In this rashion, each spacecrart mode is coupled with I-launch vehicle modes one at a time. To derive a bound on the total solution in the original $(S+L)$ mathematical space, an explicit solution in the form of spacecraft modal response time history is first derived for the pair of modes in a typical subspace. The explicit form of this solution is derived here, and is based on the idealized modal forcing function just discussed. Furthermore, because the spacecraft member loads are the ubjective of the analysis, and since these are proportional to the generalized modal displacements, the response quantities used here are generalized modal displacements, $q_{s \ell}(t)$, from which an expression of the bound $Q_{s \ell}$ of $q_{s \ell}(t)$ is then derived.

(2) Numerical computations are made first to establish the bounas, $Q_{s l}$, on each of the $(S \times L)$ discrete modal responses. Each bound $Q_{S \ell}$ corresponds to one of the $(S \times L)$ subspaces. Tn account for unknown design tolerances and variations in the structural model idealization, worst cases can be provided by allowing realistic possible tuning between each spacecraft mode and its nearest launch vehicle mode, and by scaling the entire frequency spectrum of the launch vehicle with respect to that of the spacecraft.

(3) Next, a bound in the total spacecraft modal response, $Q_{s}$, is constructed by sumation over all the discrete L-bounds for that, spacecraft mode. The summation can be over 
absolute values, or in a root-sum-square sense that can also be welghted to account for phasing.

(4) Finally, spacecraft member loads are obtained by adding the contributions of all spacecraft modes, efther in absolute value or in a root-sum-square sense.

In the procedure outlined above, steps (1) and (2) derive expressions for bounds on the discrete response, $Q_{s l}$, in each of the $(S \times L)$ modal subspaces, while steps (3) and (4) construct a bound on the complete solution in the total $(S+L)$ spece from the discrete $Q_{s l}$ bounds. In this manner, much computational effort is sared over the usual transient analysis.

The significant improvements or differences from Reference 1-2 are:

(1) Idealization of the actual forcing function by an impulse permits a simple evaluation of the shock spectra peaks. other forms of forcing function idealizations could also be dealt with in the least square sense.

(2) The shock spectra matching is applied to the modal - rather than the physical - degrees of freedom. This gives greater flexibility and more generality in the application of the procedure.

(3) The modal displacement spectra, $Q_{s \ell}$, is derived for an arbitrary pair of coupled modes, a launch vehicle mode and a spacecraft mode, regardless of the proximity of their associated natural frequencies. Thus, the a priori selection of a specific launch vehicle mode on the basis of its relative significance in pairing with a given spacecraft mode is not required.

(4) The entire treatment is believed to lend itself more readily to an automated spacecraft loads analysis that is substantially less dependent upon the analyst's intuitive ability. 



\section{SECTION II}

BASIC EQUATIONS

\section{A derivation of the equations of motion of a damped linear} structur. 1 system under the action of prescribed forces $\left\{F_{j}(t)\right\}$ and prescribed rigid body accelerations $\{\ddot{R}(t)\}$ :an be found in the literature (Reference $2-1$ ). For each normal mode $n=1,2, \cdots, N$, the equations may be written in the following form:

$$
M_{n n}\left(\ddot{q}_{n}+2 \xi_{n} \omega_{n} \dot{q}_{n}+\omega_{n}^{2} q_{n}\right)=\left\langle\phi_{n j}>\left\{F_{j}\right\}-<M_{n R}>\{\ddot{R}\}\right.
$$

where,

$$
\begin{aligned}
\ddot{R}= & \text { prescribed rigid body base motion } \\
q_{n}= & \text { generalized modal courdinates that are related to the } \\
& \text { physical degrees-of-freedom, }\{u\} \text {, by the normal modes, }\left[\phi_{n}\right], \\
& \text { such that }\left\{u_{j}\right\}=\left[\phi_{j n}\right]\left\{q_{n}\right\} \\
\left\{\phi_{n}\right\}= & \text { normal mode shape associated with } q_{n} ; \text { it is designated by } \\
& \left\{\phi_{j n}\right\} \text { when evaluated at the } j{ }^{\text {th }} \text { physical degree-or-freedom } \\
\xi_{n}= & \text { percent of critical modal damping for the } n{ }^{\text {th }} \text { mode } \\
\omega_{n}= & \text { natural frequency of the system, associated with } q_{n},\left\{\phi_{n}\right\}, \\
& \text { and } \xi_{n} \\
\left\langle M_{n R}>=\right. & \text { mass coupling between elastic and rigid body modes } \\
M_{n n}= & \text { elements of the diagonal modal mass matrix whose normalization } \\
& \text { is subsequently chosen so that } M_{n n}=1 \text { for all modes } \\
\left\{F_{j}\right\}= & \text { time variable vector of forces applied at an arbitrary location } \\
& j \text { due to an arbitrary event }
\end{aligned}
$$





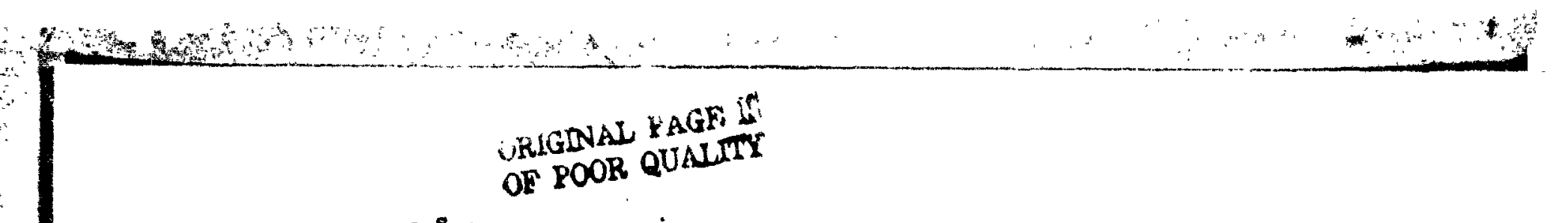

in which $\left[\mathrm{m}_{11}^{\mathrm{s}}\right]$ is the contribution of each mode $s$ to the rigid mass matrix relative to the interface 1 . The following relationship holds for each mode $\mathrm{s}$ :

$$
\left.\left[m_{i 1}^{s}\right]=\left\{m_{i s}\right\}<m_{s i}\right\rangle
$$

Rather than including all spacecraft modes, only a truncated or selected set of modes $S<T$ are usually retained. Such truncation or selection may be done by including a residual rigid mass $\left[M_{i i}\right]_{\text {RES }}$ defined by (Reference 2-2):

$$
\left[M_{i i}\right]_{R E S}=\left[M_{i i}\right]-\sum_{s=1}^{s}\left[m_{i i}^{s}\right]
$$

where $\left[M_{i i}\right]$ is the total rigid body mass matrix when all $T$ modes are considered;

$$
\left[\mathrm{M}_{i \mathrm{i}}\right]=\left[\mathrm{m}_{\mathrm{is}}\right]\left[\mathrm{m}_{\mathrm{si}}\right]
$$

Therefore, Equation (6) for $\left\{F_{i}\right\}$, can in general, be replaced by:

$$
\left\{F_{i}\right\}=\left[M_{i i}\right]_{R E S}\left\{\ddot{R}_{i}\right\}+\left(\sum_{s=1}^{s}\left[m_{i i}^{s}\right]\right)\left\{\ddot{R}_{i}\right\}+\left[m_{i s}\right]\left\{\ddot{q}_{S}\right\}
$$

Iu Fquation (8), the neglected modes arr iepresented by their equivaient rigid mass, regardless whether their frequencies are higher or lower then the range in question. In this manner one is able to consider the elastic mass contributicni of as few rir as many spacecraft modes as desirahle, with the remainine modes contributing, to the residual rigid mass. A greater number of elastic modes result in a smailer residual rigid mass matrix, which approaches zero when al] elastic modes have been included. From a modeling point of view, such 
a residual mass watrix can also heve a wide range of applications in representing the mass characteristics of part or all of the spacecrart during the varlous cycles of loads analys18. In particular, the residual mass term allows the analyst to use directly the modes of the launch vehicle loaded by a rigid mass.

For S-spececraft and L-launch vehicle elastic modes, Equations (2) and (3), along with their companion expressions $(4),(5)$, and (8) result in $(S+L)$ coupled equations describing the motion of the composite spacecraft-launch vehicle system. Instead of the commonly used transient analysis for the solution of this system of equations, a generalization of the shock spectra concept will be developed next, with the aim of obtaining a bound on the solution rather than its detailed time history. 
SECTION III

GENERALIZED MODAL DISPLACEMENT SPECTRA

\section{A. IDEALIZED EQUATTONS}

In a full transient analysis of the coupled (S $+L$ ) Equations (2) and (3), all modes are simultaneously considered, and the solution is sought as time histories of the response quantities of interest. Since bounds rather than time histories are needed for desifn, much computational effort can be saved by introducing mathematical idealizations that allow a relatively simple estimate of the bounds. This is achieved here when the entire $(S+L)$ mathematical space of modal coordinates is idealized by an array of nested $(S \times L)$ mathematical discrete subspaces. In each subspace, a spacecraft mode $s$ is coupled with a launch vehicle mode $\ell$, thus giving rise to $(S \times L)$ possible pairings. A bound on the true solution in the uriginal $(S+L)$ mathematical space is established by summation over the individual discrete bounds in the ( $S \times I)$ subspaces.

Because the spacecraft member loads are the object of the analysis here, and since these are proportional to the gencralized modal displacements $q_{s}$, bounds on the spacecraft member loads are expressed in terms of the bounds, $Q_{S \ell}$, of the generalized modal displacements for exch subspace. A typical generalized modal aisplacement bound, $Q_{s \ell}$, is derived here for a typical subspace in which one spacecraft mode $q_{s}$ is coupled with one launch vehicle mode $q_{\ell}$. For this purpose, Equations $(4),(5)$, and (8) are solved together to yield:

$\left.\begin{array}{l}\left\{\ddot{\mathrm{B}}_{i}\right\}=\left([I]+\left[\psi_{i i}\right]\left[M_{i i}^{s}\right]\right)^{-1}\left(\left[\psi_{i j}\right]\left\{F_{j}\right\}+\left\{\phi_{i \ell}\right\} \ddot{q}_{\ell}-\left[\psi_{i i}\right]\left\{m_{i s}\right\} \ddot{q}_{s}\right) \\ \left\{F_{i}\right\}=\left([I]+\left[M_{i i}^{s}\right]\left[\psi_{i i}\right]\right)^{-1}\left(\left[M_{i i}^{s}\right]\left[\psi_{i j}\right]\left\{F_{j}\right\}+\left[M_{i i}^{s}\right]\left\{\phi_{i l}\right\} \ddot{q}_{\ell}+\left\{m_{i s}\right\} \ddot{q}_{s}\right)\end{array}\right\}(9)$ 



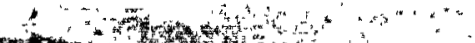


In which the following additional definitions have been used:

$$
\begin{aligned}
\mu_{s l} & =\frac{m_{s l}}{1+M_{s l}} \\
\omega_{1} & =\frac{w_{l}}{\sqrt{1+M_{s l}}} \\
\xi_{1} & =\frac{\xi_{l}}{\sqrt{1+M_{s l}}} \\
\omega_{2} & =\omega_{s} \\
\xi_{2} & =\xi_{s} \\
F_{\ell}(t) & =\left\langle\phi_{\ell j}>\left\{F_{j}(t)\right\}\right. \\
q_{s} & =\left(\frac{<m_{s i}>\left\{\phi_{1 \ell}\right\}}{1+M_{s l}}\right) x_{2}=\left(\frac{1 m_{s l}}{1+M_{s l}}\right) x_{2} \\
q_{\ell} & =\left(\frac{1}{I+M_{s l}}\right) x_{1}
\end{aligned}
$$

B. MODAL DISPLACEMENT SPECTRA

The pair of modally coupled equations in Equation (13) describe the motion in any of the idealized ( $\times L$ ) subspaces where a spacecraft mode is coupled with a launch vehicle mode. A bound on the spacecraft generalized modal displacernent in such subspace is derived in this section from an explicit time-dependent solution of Equation (13), in which the actual launch vehicle modal forcing function $F_{\ell}(t)$ is modeled 



$$
\begin{aligned}
x_{1}(t)= & \frac{v_{0 l}}{\left(\Omega_{2}^{2}-\Omega_{1}^{2}\right)}\left[\frac{\Omega_{2}^{2}\left(1-\Omega_{1}^{2}\right)}{\bar{\omega}_{n 1}} e^{-\xi_{n 1} \omega_{n 1} t} \sin \bar{\omega}_{n 1} t\right. \\
& \left.-\frac{\Omega_{1}^{2}\left(1-\Omega_{2}^{2}\right)}{\bar{\omega}_{n 2}} e^{-\xi_{n 2} \omega_{n 2} t} \sin \bar{\omega}_{n 2} t\right] \\
x_{2}(t)= & \frac{R^{2} v_{0 \ell}}{\left(\Omega_{2}^{2}-\Omega_{1}^{2}\right)}\left[\frac{1}{\bar{\omega}_{n 1}} e^{-\xi_{n 1} \omega_{n 1} t} \sin \bar{\omega}_{n 1} t\right. \\
& -\frac{1}{\bar{\omega}_{n 2}} e^{-\xi_{n 2} \omega_{n 2} t} \sin \bar{\omega}_{n 2} t
\end{aligned}
$$

The following parameters have been used in Equation (15):

$$
\begin{aligned}
& \omega_{n 1}=\omega_{2} \Omega_{1} \text {, } \\
& \omega_{n \varepsilon}=\omega_{2} \Omega_{2} \\
& \Omega_{1}=\Omega_{0}-\frac{\Delta \Omega}{2} \\
& \Omega_{2}=\Omega_{0}+\frac{\Delta \Omega}{2} \\
& \Omega_{0}=\frac{1}{2} \sqrt{(R+1)^{2}+\mu_{s l}} \\
& \Delta \Omega=\sqrt{(R-1)^{2}+\mu_{s \ell}} \\
& R=\frac{\omega_{1}}{\omega_{2}}=\Omega_{1} \Omega_{2} \\
& \bar{\omega}_{n 1}=\omega_{n 1} \sqrt{1-\xi_{n 1}^{2}}, \quad \bar{\omega}_{n 2}=\omega_{n 2} \sqrt{1-\xi_{n 2}^{?}} \\
& \xi_{n 1}{ }_{n 1}+\xi_{n 2} \omega_{n 2}=2 \omega_{2} \beta, \quad \xi_{n 1} \omega_{n 1}-\xi_{n 2}{ }_{n 2}=2 \omega_{2} \theta \\
& \beta=\frac{1}{2}\left[\xi_{I} R+\left(I+\mu_{s l}\right) \xi_{2}\right] \\
& \theta=\left[\frac{\xi_{1} R\left(1-\Omega_{1}^{2}\right)^{2}+\mu_{s l_{2} \xi_{2} \Omega^{4}}}{\left(1-\Omega^{2}\right)^{2}+\mu_{s l}}-\frac{\xi_{1} R+\left(1+\mu_{s l}\right) \xi_{2}}{2}\right]
\end{aligned}
$$


The objective is to determine the maximum value of the displacement $x_{2}(t)$ relative to the "base motion" $x_{1}(t)$. The maximum value of $x_{2}(t)$ is called the "generalized" displacement shock spectra since the "base acceleration" $\ddot{x}_{1}(t)$ is alloved to be affected by $\ddot{x}_{2}(t)$, unilke the aimple traditional shock spectre concept. To derive relatively simple expressions requiring minimal numerical calculation for the generalized modal displacement spectra, further mathematical simplifications will be introduced in Bquations (15) and (16). These simplifications were tested and found accurate when the results below were compared with the results of the analog solution of Appendix A.

First, since $\xi_{n 1}^{2}$ and $\xi_{n 2}^{2}$ are much smaller than unity, the approximation

$$
\begin{aligned}
& \text { for } R \neq 0: \quad \xi_{n 1} \doteq \xi_{n 2} \doteq \xi_{n}=\frac{\xi_{n 1}+\xi_{n 2}}{2}=\frac{1}{\omega_{2} R}\left[\Omega_{0} \beta+\frac{\Delta \Omega l}{2} 0\right] \\
& \text { for } R=0: \quad \xi_{n}=\frac{\xi_{2}}{2}
\end{aligned}
$$

is used for the damped natural frequencies $\bar{\omega}_{n 1}$ and $\bar{\omega}_{n 2}$, so that:

$$
\begin{aligned}
& \bar{\omega}_{n 1} \doteq \omega_{2}\left(\rho_{1}-\rho_{2}\right) \\
& \bar{\omega}_{n 2} \doteq \omega_{1}\left(\rho_{1}+\rho_{2}\right)
\end{aligned}
$$

where

$$
\begin{aligned}
& \omega_{2} \rho_{1}=\omega_{2} \Omega_{0} \sqrt{1-\xi_{n}^{2}}=\text { damped center frequency } \\
& { }^{\prime \prime} \cdot{ }^{\prime} \rho_{2}=\omega_{2} \frac{\Delta \Omega}{2} \sqrt{1-\xi_{n}^{2}}=\text { damped beat frequency }
\end{aligned}
$$


Note that the approximation of Equation (17) is not used for the exponential part of the solution. Also note $\Delta \Omega / 2 n_{0}=p_{2} / p_{1}$.

Furthermore, according to Equation (16), $\theta \ll \beta$. In fact, $\theta$ tends to be exceedingly small in the neighborhood or $R=1$. Therefore, it is sufficient to retain only the first order approximation for $e^{\theta t}$, i.e., $e^{\theta t}+1+\theta t$, and correspondingly $e^{-\theta t}=1-\theta t$. This approximation, along with that of Equation (17) and some trigonometric transformations, lad to the following simplified expression for the relative displacement $x_{2}(t)$ for the spacecraft mode:

$$
x_{2}(\tau) \div-w_{1} v_{0 \ell}\left[D_{1}(\tau)+D_{2}(\tau)\right], \quad \tau=w_{2} t
$$

where

$$
\begin{aligned}
& D_{1}(\tau)=\frac{1}{2 \omega_{2}^{2} \rho_{2}}\left|\frac{\rho_{2}}{\rho_{1}}-\theta \tau\right| e^{-\beta \tau} \sin \rho_{1} \tau \cos \rho_{2} \tau \\
& D_{2}(\tau)=\frac{-1}{2 \omega_{2}^{2} \rho_{1}}\left|\frac{\rho_{1}}{\rho_{2}}-\theta \tau\right| e^{-\beta \tau} \cos \rho_{1} \tau \sin \rho_{2} \tau
\end{aligned}
$$

For $R \neq 0:$ since $\rho_{2}$ is smaller than $\rho_{1}$, an accurate estimate of the maximum value of the relative displacement $x_{2}(t)$ of Equation (20) is provided by the discrete maxima $D_{\max }$ of the simple sinusoid, where $\cos \rho_{1} \tau$ and $\cos \rho_{2} \tau$ are either zero or unity, (see Figure 3-1). When $D_{1}(\tau)$ approaches its maximum $D_{1 \max }, D_{2}(\tau)$ approaches zero, and vice versa. Therefore, the maximum relative displacement $D_{\max }$ is given by the largest of $D_{1 \max }$ or $D_{2 \max }$ :

$$
\begin{aligned}
& D_{1 \text { max }}=\frac{1}{2 \omega_{1}^{2} \rho_{2}}\left(\frac{\rho_{2}}{\rho_{1}}-\theta \tau\right) e^{-\beta \tau} \sin \rho_{1} \tau \\
& D_{2 \max }=\frac{1}{2 \omega_{2}^{2} \rho_{1}}\left(\frac{\rho_{1}}{\rho_{2}}-\theta \tau\right) e^{-\beta \tau} \sin \rho_{2} \tau
\end{aligned}
$$



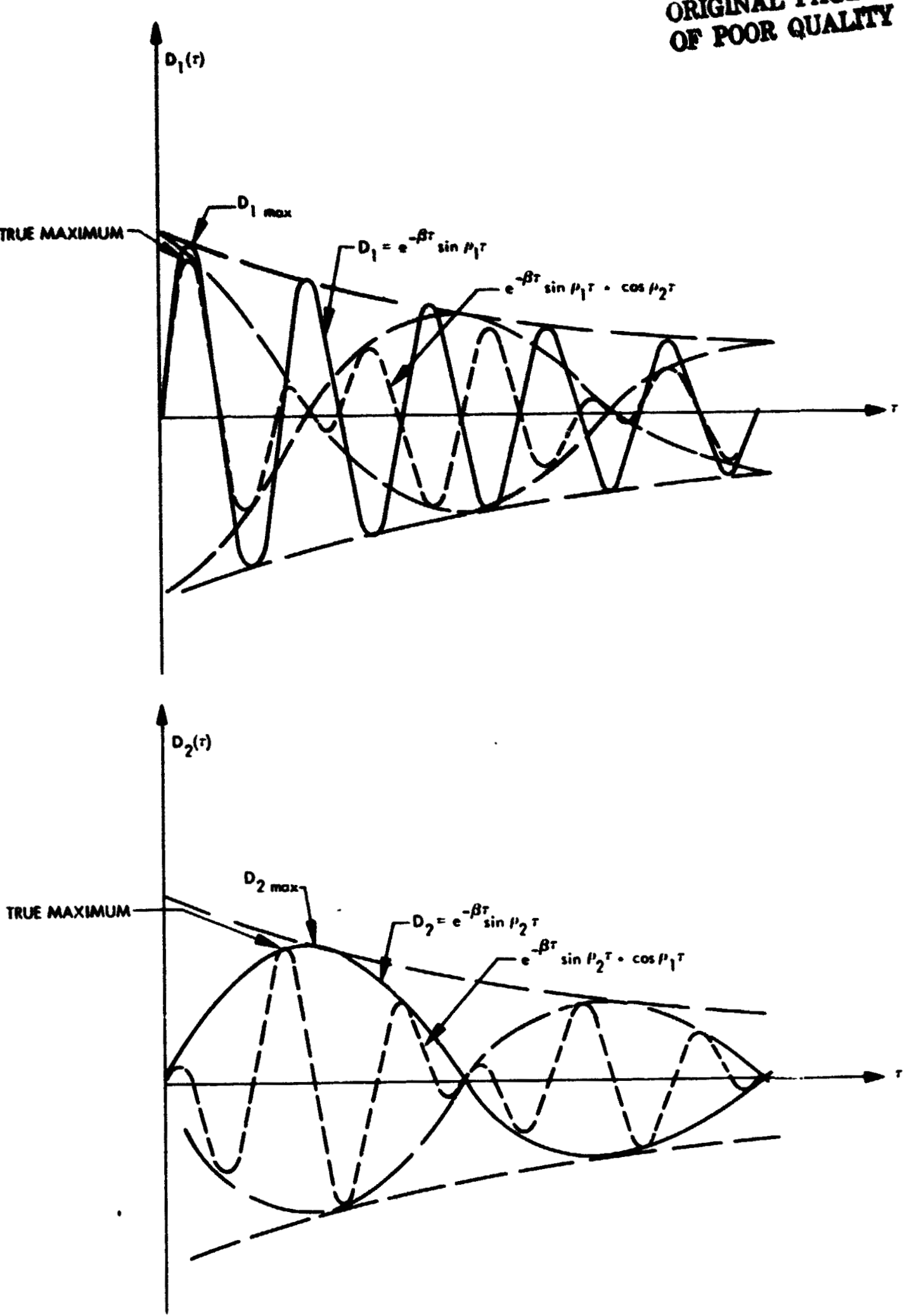

Figure 3-1. Graphic Representation of $D_{1}(t), D_{2}(t)$, and

Their Approximate Maxima $D_{1 \max }$ and $D_{2 \max }$ 
where $t$ is determined as the smallest root of the characteristic equations

$$
\begin{aligned}
& \text { for } D_{1 \max }: \quad \tan p_{1} \tau=\frac{\rho_{1}}{\beta} \frac{\left[1-\left(\rho_{1} / \rho_{2}\right) \theta \tau\right]}{\left[1+\left(\rho_{1} / \rho_{2}\right) \frac{\theta}{\beta}(1-\beta \tau)\right]} \\
& \text { for } D_{2 \max }: \quad \tan \rho_{2} \tau=\frac{\rho_{2}}{\beta} \frac{\left[1-\left(\rho_{2} / \rho_{1}\right) \theta \tau\right]}{\left[1+\left(\rho_{2} / \rho_{1}\right) \frac{\theta}{\beta}(1-\beta \tau)\right]}
\end{aligned}
$$

The definitions introduced in Equation (14) and the equalities of Equations (21) and (22) are now combined to give the generalized mocial displacement spectra, $Q_{s \ell}$ :

$$
Q_{s l}=\left|\frac{\omega_{1} v_{O \ell} \sqrt{m_{s l}}}{\left(1+M_{s l}\right)} D_{\max }\right|
$$

Equation (23) above, along with its companion expressions of Equations (21) and (22) represent a generalization of the traditional shock spectra concept since these expressions account for interactions between the oscillator and its base, i.e., between the spacecraft and launch vehicle modes. They also represent an improvement over the results of Reference 1-2 in that they hold true for an arbitrary pair of coupled modes, a launch vehicle mode and a spacecraft mode, regardless of the proximity of their respective natural frequencies. It is this fact that makes it possible in the present approach to include contributions of all launch vehicle and spacecraft modes in the computation of bounds on the modal displacements and subsequently on the member loads. It is noted here that Equation (2I) suggests that the damping effect is through the "average" damping $B$ of the spacecraft and the launch vehicle as defined in Equations (16) rather than through each damping separately. This point is explained further in Appendix A. Although derived for $R \neq 0$, Equation (23) is still valid in the limit for $R \rightarrow 0$. This is show in Appendix $D$. 


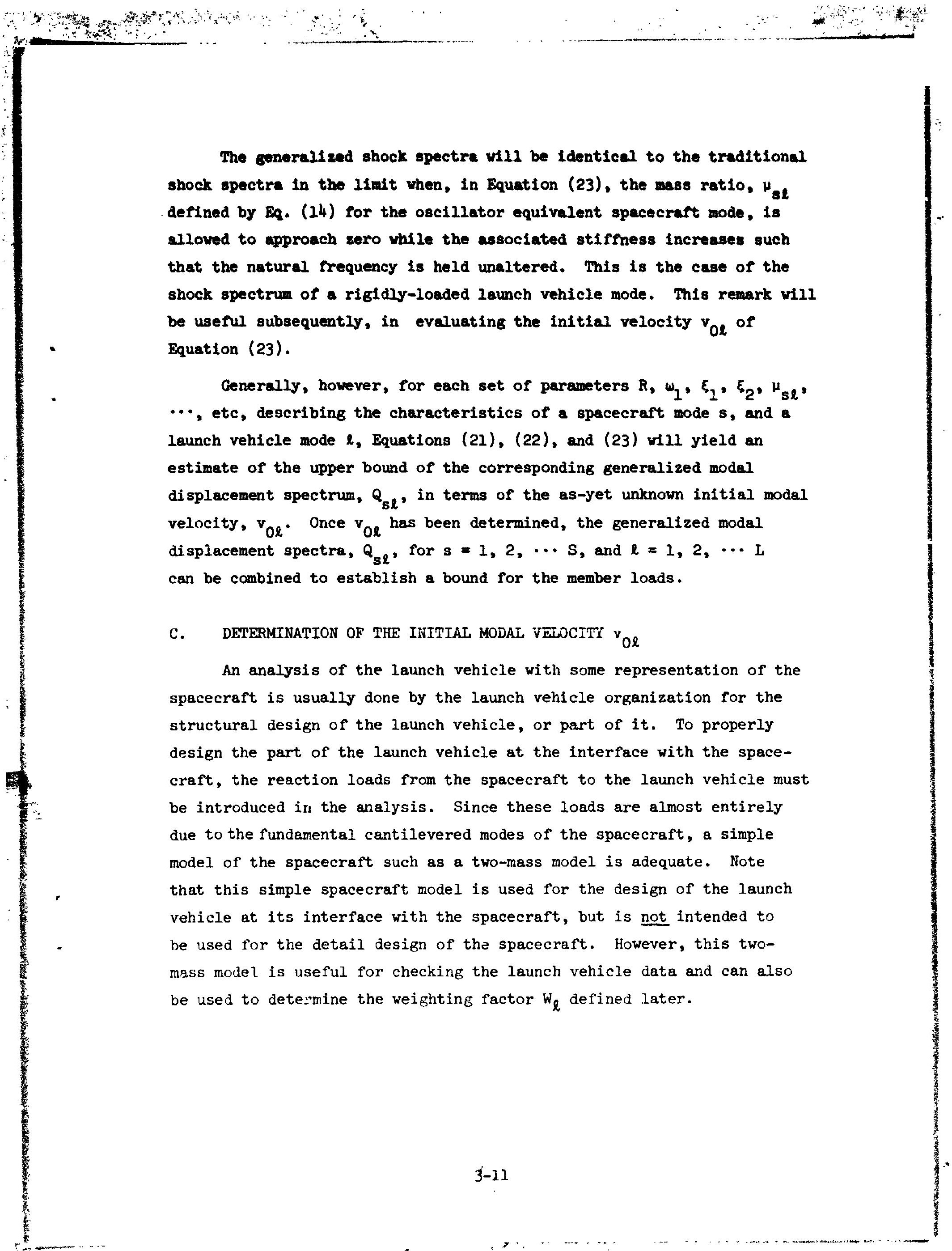




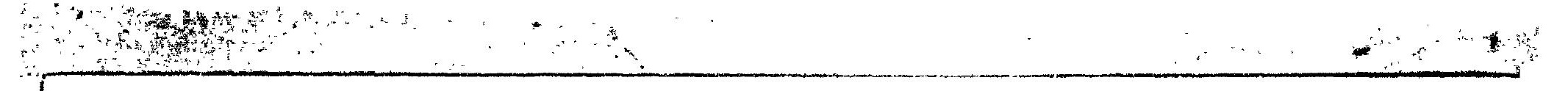

The orfiginal launch vehicle forcing functions as used for the launch vehicle analysis are given by a set of components $\left\{F_{9}(t)\right\}$ expressed in the physical coordinate system $x y z$ and applied at given locations of the launch vehtcle structure. The modal analysis done on the launch vehicle with any spacecraft or dummy provides, in effect, a new system of coordinates, 1.e., the generalized coordinates $q_{c}(t)$, that are used to express new component 3 of the forcing functions as a set of generailized forces $F_{c}(t)$. In effect, $F_{c}(t)$ is another definition of the same forcing functions $F_{j}(t)$. Therefore, one can use this set $\left\{F_{c}(t)\right\}$ to represent the forcing functions without the need of knowing the physical components, nor the location of the original forcing functions. Note that this observation holds only because an analysis of the launch vehicle with a dumny spacecraft has already been done.

Any further change of modes, if necessary, will simply change the set $\left\{F_{c}(t)\right\}$ by a modal transformation. For example, the present method requires the use of the modes of the launch vehicle loaded only by a rigid mass. Therefore, the components $\left\{F_{\ell}(t)\right\}$ of the forcing functions with respect to the rigid mass loaded launch vehicle modes are

$$
\left\{F_{\ell}(t)\right\}=\left[v^{T}\right]\left\{F_{c}(t)\right\}
$$

where $[\mathrm{V}]$ is the modal matrix that transforms the elastically loaded launch vehicle to the rigid-mass loaded one as shown in Appendix $C$.

Similarly, the generalized coordinates $\left\{\ddot{q}_{\ell}(t)\right\}$ of the rigid mass loaded launch vehicle are obtained from the coordinates $\left\{\ddot{q}_{c}(t)\right\}$ of the elastically loaded launch vehicle by Equation (c-18) or Equation (c-20) of Appendix $C$.

The unknown initial modal velocity $v_{0 l}$ can be determined from the requirement that the "actual" forcing function and the "idealized" forcing function produce identical bounds for the modal displacement spectra. Information for the "actual" forcing function can be obtained either from an initial analysis done on a simplified model of the spacecraft-launch vehicle combination, or from recorded flight response data for another spacecraft. It is believed that the minimum required 
Information is three degrees of freedom at the spacecraft-launch vehicle interface. The knowledge of more degrees of freedom improves the definition of $v_{0 l}$. In case of an available inftial analysis, the knowledge of the modal response-time histories for each mode leads to the best estimate of $\mathrm{v}_{\mathrm{Ol}}$. For the last case, the peak value of the traditional displacement shock spectra, $D_{\ell}^{P}$, is calculated for the modal acceleration response $\ddot{q}_{\ell}(t)$ of the rigid mass loaded launch vehicle as shown by Equations (28) and (29).

Finally, it should be noted that $D_{\ell}^{P}$ will need to be computed only once for each launch vehicle mode and each flight event of interest. This part of the computation will :emain the same as long as the launch vehicle and the forcing functions are not changed.

In the derivation done so far and in what follows, rigid mass loaded launch vehicle modes are used. These modes are obtained by the method of Appendix . , or directly from the launch vehicle analysis if the spacecraft was represented by a rigid mass, as may be the case for preliminary launch velıicle design.

1. Estimate of $v_{0 l}$ From the Modal Response

Assuming now that the time histories $\ddot{q}_{l}(t), \ell=1,2, \cdots, L$ for the rigidly-loaded launch vehicle are available from a previous launch vehicle analysis, and that $D_{l}^{P}\left(\omega_{2}, \xi_{2}\right)$ representing the peak of the modal displacement spectrum $D_{\ell}\left(\omega_{2}, \xi_{2}\right)$ for each mode has been evaluated, then $v_{0 l}$ can be determined by requiring that $D_{\ell}^{P}$ be equal to its counterpart, $Q_{l}^{P}$, derived from Equation (23) for $\mu_{s l}+0$. This is illustrated in Figure 3-2. To derive $Q_{\ell}^{P}$ for $R \neq 0$, the shock spectrum $Q_{Q}$ is computed for each of the launch vehicle elastic modes $l=7,8, \cdots$, L from Equation (23) in the limit as $\mu_{s \ell} \rightarrow 0$. Appendix $D$ gives the proper values of the modal shock spectra for the case when $R=0$, that is, for $\ell=1, \cdots, 6$.

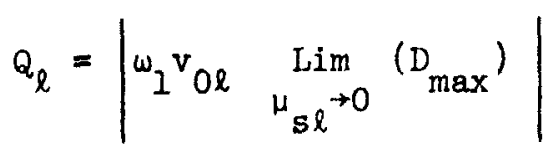




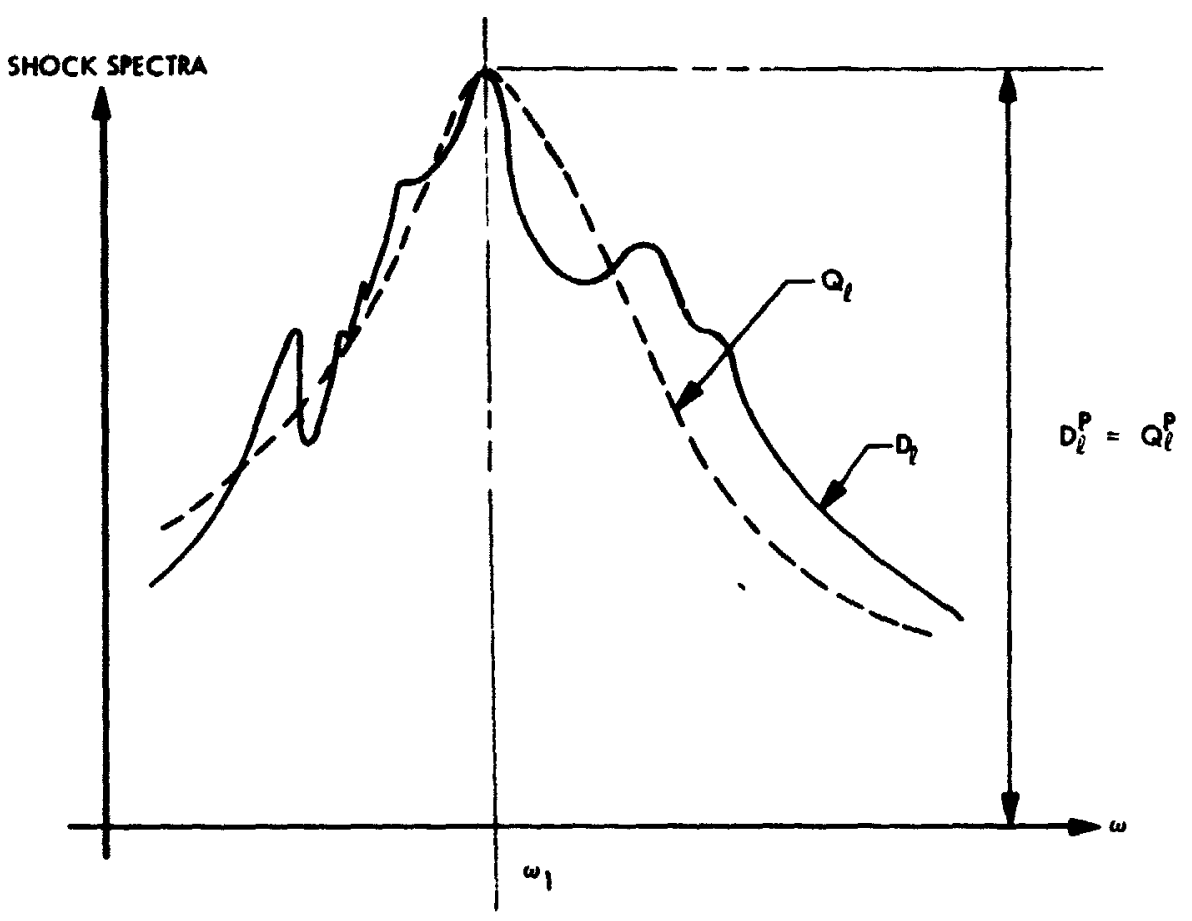

Figure 3-2. Modal Displacement Spectra $D_{\ell}$ and $Q_{\ell}$ for a Launch Vehicle Mode $\ell$ 
The quant1ty, $\underset{\mathcal{L}}{L} \operatorname{Lim}_{\mathrm{s} \rightarrow 0}\left(D_{\max }\right)$, of Equation (25) is readily evaluated by substituting the following parameters in Equations (21) and (22): as $\mu_{82} \rightarrow 0$, and $R \neq 0$

$$
\begin{aligned}
B & =\frac{1}{2}\left(\xi_{1} R+\xi_{2}\right) \\
\theta & =\frac{1}{2}\left(\xi_{1} R-\xi_{2}\right) \\
\frac{2 \Omega_{0}}{\Delta \Omega} & =(R+1) /(R-1) \\
\rho_{1} & =\frac{1}{2}(R+I) \sqrt{1-\xi_{n}^{2}} \\
\rho_{2} & =\frac{1}{2}(R-I) \sqrt{1-\xi_{n}^{2}} \\
\xi_{n} & =\frac{1}{2}\left(\xi_{1}+\xi_{2}\right)
\end{aligned}
$$

As seen from Equation (26), the displacement shock spectrum of Equation (25) is dependert upon the value of $R=\omega_{1} / \omega_{2}$. The peak value of $Q_{\ell}$, labeled $Q_{\ell}^{P}$, occurs for $R \rightarrow R^{P}$. Finding the exact value of $R^{P}$ that maximizes $Q_{\ell}$ is rather involved analytically. Only an approximate estimate will be given here.

Considering the special case when $\theta$ and $\xi_{n}^{2}$ of Equation (26) are assumed negligibly small, we lind that

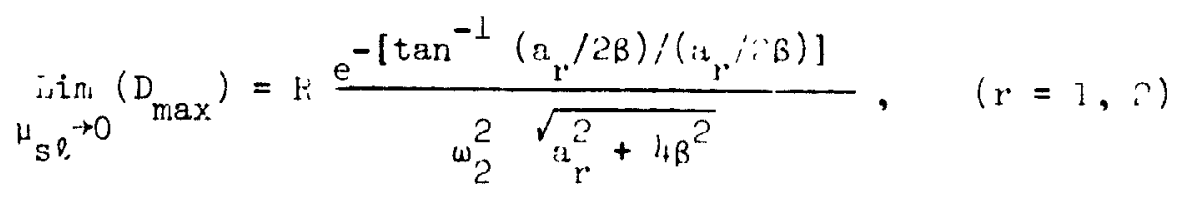


where

$$
a_{r}= \begin{cases}(R+1) & \text { for } r=1 \\ (R-1) & \text { for } r=2\end{cases}
$$

By inspection of Equation (27), it is concluded that the peak occurs for $r=2$ and $R^{P}=1$. The approximation that $\theta$ in Equation (26) is negligibly small, is of the same order as the approximation that the Imaginary part of the solution of Equation (15) is negligible, both of which are acceptable. The exact solution was evaluated from the analog simulation of Appendix $A$, in which the maximum of $D_{\max }$ occurred within better than $1 \%$ from $R=1$. For $R^{P}=1$, Equations $(25)$ and $(26)$ yield the following expression for the initial modal velocity and for $\omega_{1} \neq 0$ :

$$
\omega_{1} v_{0 l}=e \omega_{1}^{2}\left(\xi_{1}+\xi_{2}\right) D_{l}^{P}\left(\omega_{2}, \xi_{2}\right)
$$

where $e=2.718$

Instead of the modal displacement peak $\mathrm{D}_{\ell}{ }^{\mathrm{P}}\left(\omega_{2}, \xi_{2}\right)$, the modal acceleration peak $\mathrm{A}_{\ell}^{\mathrm{P}}\left(\omega_{2}, \xi_{2}\right)$ may be used. At the peak of the displacement, the two quantities are related by $\omega_{2}^{2} D_{l}^{P}\left(\omega_{2}, \xi_{2}\right)=A_{l}^{P}\left(\omega_{2}, \xi_{2}\right)$. Thus, the following altemate expression for $v_{0 l}$ is used, noting that $w_{1}=w_{2}$ :

$$
\omega_{1} v_{O \ell}=e\left(\xi_{1}+\xi\right) A_{\ell}^{P}\left(w_{2}, \xi\right)
$$

for $\omega_{1} \neq 0$ and where $\xi$ is the damping used in calculating the acceleration shock spectra $A_{\ell}$ : $f \ddot{q}_{\ell}(t)$.

As shown in Appendix D, for $\omega_{1}=0$ Equation (29) becomes $\left(\omega_{\perp} v_{O \ell}\right)_{\omega_{l} \rightarrow 0}=A_{\ell}^{P}$

2. Estimate of $v_{0 \ell}$ From Physical Interface Response In the absence of availability of $\ddot{q}_{c}(t)$, a set of initial modal velocities $v_{0 \ell}$ can also be obtained in a manner similar to that of 
Reference $3-1$. It is assumed that $t^{*}$ - tine histories from an analysis or flight data of an $N_{0}$ degrees of freedom at the launch vehicle/spacecraft interface are known for another snacecraft or a dummy one. The shock spectra $s_{1}\left(1=1,2, N_{0}\right)$ for the $N_{0}$ degrees of freedom are calculated, as typically represented by the solld line of Flgure 3-3.

Next it is assumed that the mode shapes $\phi_{n}$ of the structure corresponding to the avallable interface acceleration are also known. Then each mode is given an initial velocity $v_{O_{n}}$ and the total interface acceleration is calsulated from:

$$
\ddot{R}_{i}(t)=-\sum_{n=1}^{N} \omega_{n} v_{0 n} \phi_{n} e^{-\xi_{n} \omega_{n} t}\left(a_{n} \sin \omega_{d} t+b_{n} \cos \omega_{d} t\right)
$$

where

$$
\begin{aligned}
& a_{n}=\left(1-2 \xi_{n}^{2}\right) / \sqrt{1-\xi_{n}^{2}} \\
& b_{n}=2 \xi_{n} \\
& \omega_{d}=\omega_{n} \sqrt{1-\xi_{n}^{2}}
\end{aligned}
$$

The value of $v_{0 n}$ can be determined by trial and error so that the shock spectra of $\ddot{R}_{i}(t)$ above, represented by the dashed line in Figure 3-3, envelopes the shock spectra of the real response. Since only a small number of vehicle modes are significantly contributing to $\ddot{R}_{i}$, one can choose a set of modes $\bar{N}<N$ to simplify the calculations. The important criteria is that the $v_{\text {on }}$ and the mode retained give an envelope of the real shock spectra.

Finally, the initial modal velocity $v_{0 \ell}$ for the rigid mass loaded launch vehicle is obtained by the transformation of Equation (3i), where a bound for $v_{0 l}$ will be obtained by taking root sum square values, since the sign of $v_{0 n}$ is not retained. 


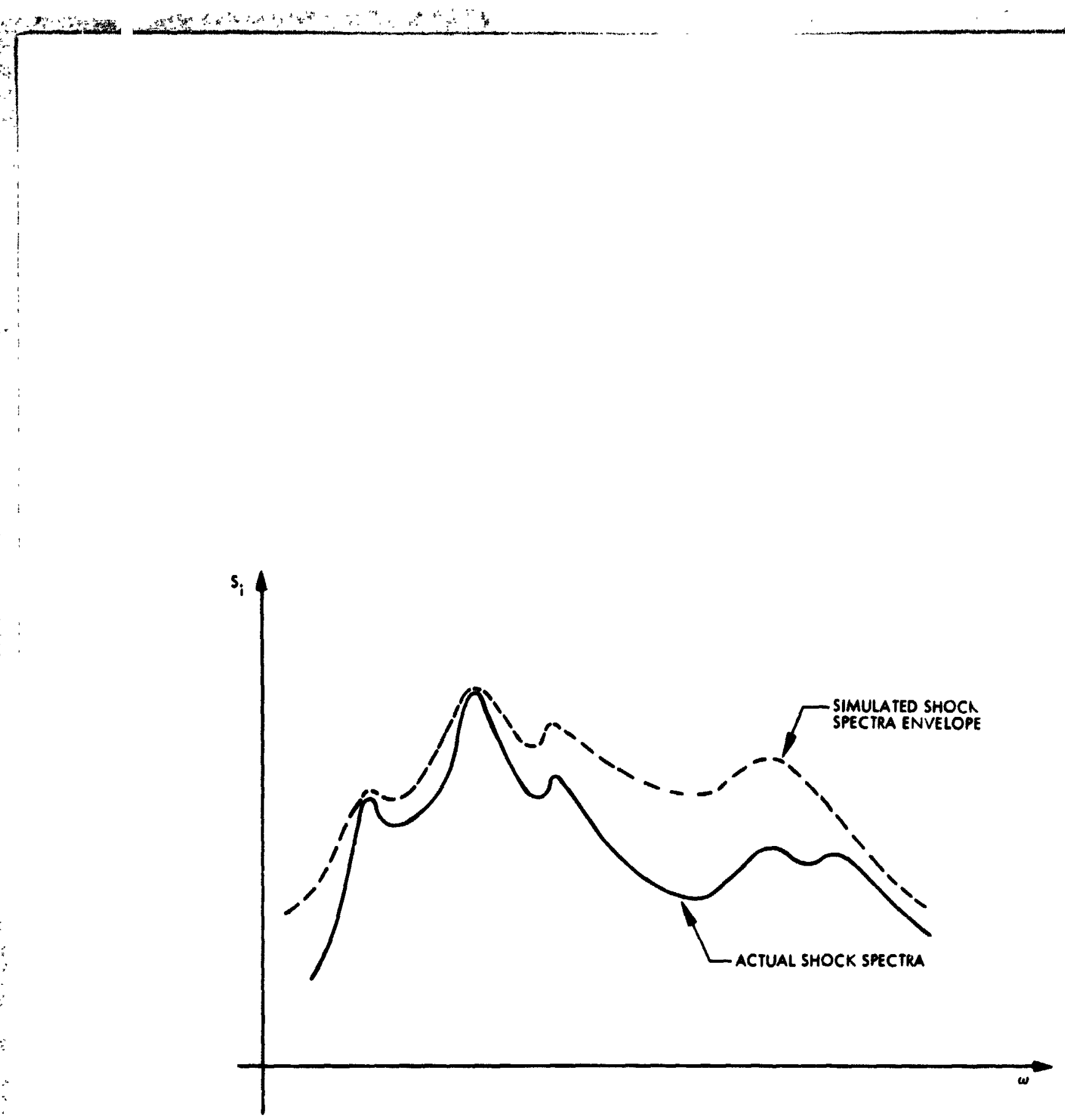

Figure 3-3. Shock Spectra Matching and Envelope 


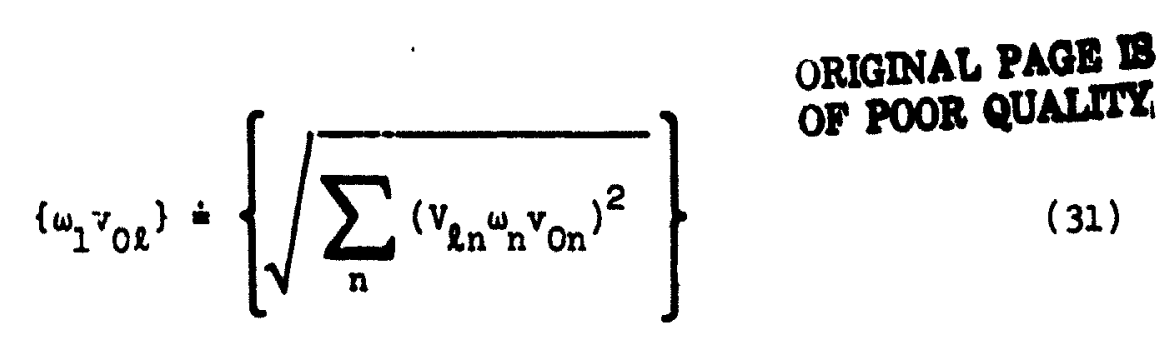

Note that this method is only a fall-back method, and that the modal response method of the previous section is to be preferred.

D. TUNING

During the early stages of design, the spacecrart and launch vehicle modes and frequencies are obtained from analyses that usually contain a large degree of uncertainty. To account for such uncertainties, one may introduce an artificial tuning between the spacecrafi and launch vehicle modes. Unlike other methods, the present approach makes artificial tuning possible and easy to implement because Equation (23) is inexpensive to evaluate and is valid for any pair of spacecraft and launch vehicle modes, regardless of the proximity of their respective frequencies.

Two forms of artificial tuning have been identified: global and local. In global tuning, the entire spectrum of launch vehicle frequencies is incrementally caled in eithe: direction $\cdots: \because,: 50$ the spacecraft frequency spectrum. For each increment, a giobal respnrse

$$
\bar{Q}=\sqrt{\sum_{\ell} \sum_{s}{ }^{2} s \ell}
$$

is computed and used as a measure for determinine, the worst case for design purposes. In this scheme, tuning is achieved by finding the amount of relative scaling that maximizes $\vec{Q}$. Clearly, limits on the allowable relative scaling must be selected in advance, and the search for the muximum $\bar{Q}$ conducted within these limits.

In the local tuning, the response is maximized for each spacecraft mode, one at a time. This is achieved by allowing the necrest launch vehicle irequency to coincide with that of the spacecraft frequency 
under consideration, (1.e., $R \rightarrow 1$ ) provided that the two were originally separated by no more than a preselected amount. Other schemes for tuning can be also devised. 


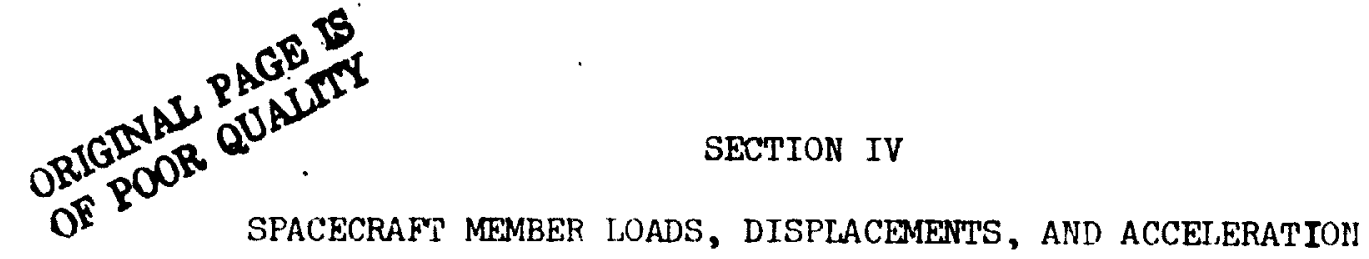

A. MEMBER LOADS

When the time histories of the spacecraft generalized modal displacement $q_{s}(t)$ are determined as a result of a transient analysis, one can express the vector of the spacecraft modal member loads, $\left\{f_{\text {as }}(t)\right\}_{A}$, for each member $A$ due to motion in mode $s$ in the general form (Reference $2-1$ ):

$$
\left\{f_{a s}(t)\right\}_{A}=\left[c_{a b}\right]\left\{\phi_{b s}\right\} q_{s}(t)
$$

where

$$
s=1,2, \cdots, s
$$

In addition, the total load vector due to contributions of all spacecraft modes is

$$
\left\{f_{a}(t)\right\}_{A}=\sum_{S=1}^{S}\left\{f_{a s}(t)\right\}_{A}
$$

where

$$
\begin{aligned}
{\left[c_{a b}\right]_{A}=} & \text { matrix of force coefficients, whose elements are the } \\
& a^{\text {th }} \text { force conponent in member A due to a unit displace- } \\
& \text { ment in the } b^{\text {th }} \text { degree of freedom } \\
\left\{\phi_{b s}\right\}= & \text { as before, spacecraft mode shape at the } b^{\text {th }} \text { degree- } \\
& \text { of-freedom } \\
{\left[c_{a b}\right]\left\{\phi_{b s}\right\}=} & \text { is recognized as the vector of modal stresses for mode } s .
\end{aligned}
$$

In the present approach, the time histories of the spacecraft generalized modal displacements $q_{s}(t)$ in Equations $(32)$ and $(33)$ are 
not computed for the intire $(S+L)$ mat " atical space. Instead, they are replaced by an estimate of their upper bound, $Q_{s}$, so that the correspondin;s bound on the spacecraft modal member loads, $\left\{\mathrm{F}_{\text {as }}\right\}_{A}$ is uritten ansiogous to Equation (32) in the form:

$$
\left[F_{a s}\right)_{A}=\left[c_{a b}\right]_{A}\left\{\phi_{b s}\right\} Q_{s}
$$

The bound, $Q_{s}$, for the spacecraft generalized modal displacement is expressed in terms of the discrete modal displacement spectra, $Q_{s l}$ of Equation (23) for each of the $(S \times L)$ discrete mathematical subspaces. Since each $Q_{s l}$ results from coupling between a spacecrait mode $s$ and only one launch vehicle mode, and since complete representation of the launch vehicle includes more than one mode, say $L$ modes, contributions due to all $L$ modes should be included. Clearly, because Equation (23) does not contain information regarding time-phasing among modes, only a bound can be computed, and is provided by summation over absolute values or in the root-sum-square sense.

Thus, $Q_{s}$ is estimated by

$$
Q_{s}=\sum_{l=1}^{L}\left|Q_{s l}\right|
$$

or

$$
Q_{s}=\sqrt{\sum_{l=1}^{L} Q_{s l}^{2}}
$$

or

$$
Q_{s}=\sqrt{\sum_{\ell=1}^{L}\left[w_{\ell}\left(w_{s}, w_{\ell}\right) Q_{s \ell}\right]^{2}}
$$




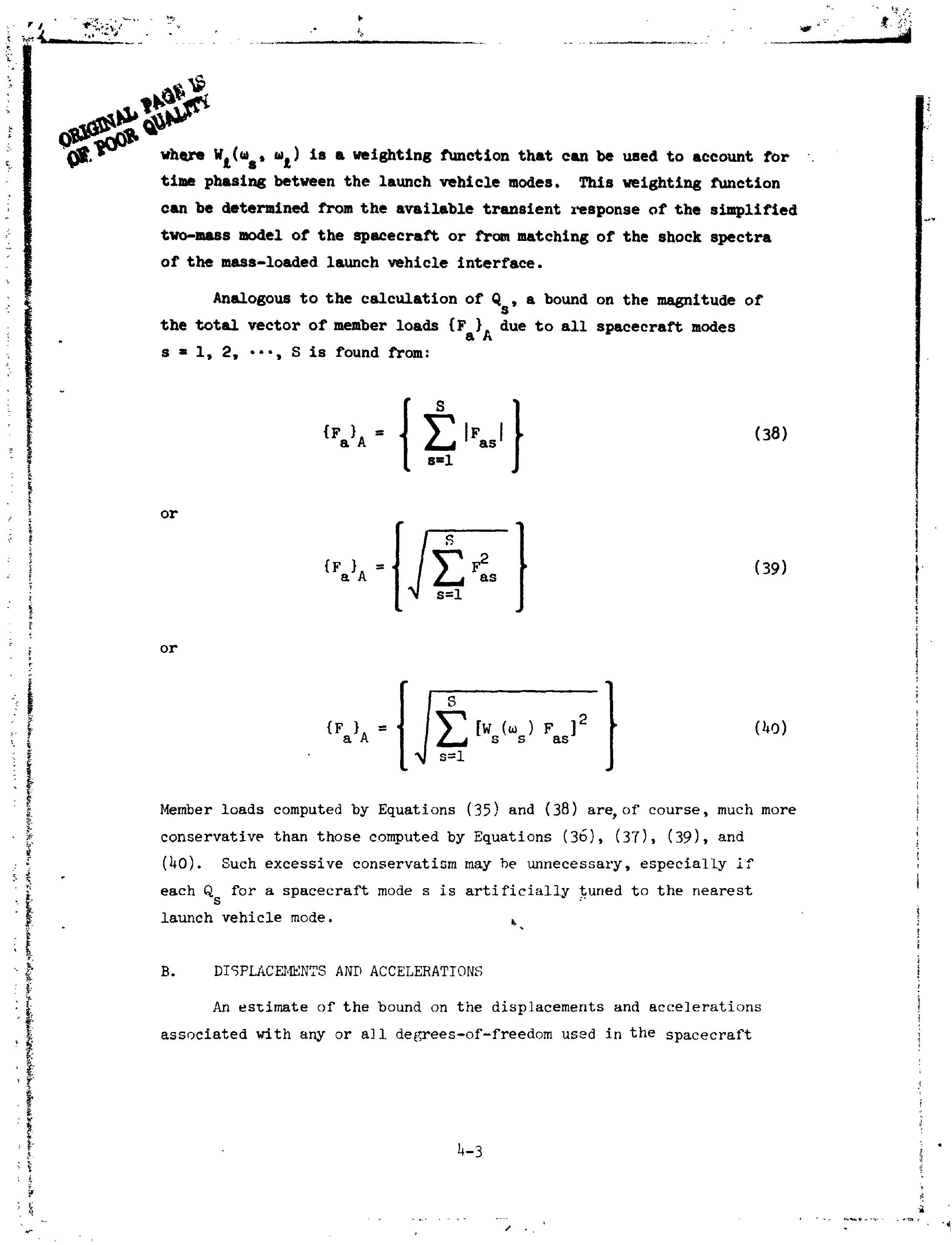



Similarly, for the accelerations,

$$
\left[A_{b}\right\}=\left\{\sum_{s=1}^{s}\left|A_{b s}\right|\right\}
$$

or

$$
\left\{A_{b}\right\}=\left\{\sqrt{\sum_{s=1}^{S} A_{b s}^{2}}\right\}
$$

or

$$
\left\{A_{b}\right\}=\left\{\sqrt{\sum_{s=1}^{S}\left[w_{s}\left(w_{s}\right) A_{b s}\right]^{2}}\right\}
$$

\footnotetext{
C. FLOW DIAGRAM

Figure 4-1 summarizes the different steps taken from the launch vehicle data to the computation of bounds for the member loads, displacements, and accelerations.
} 


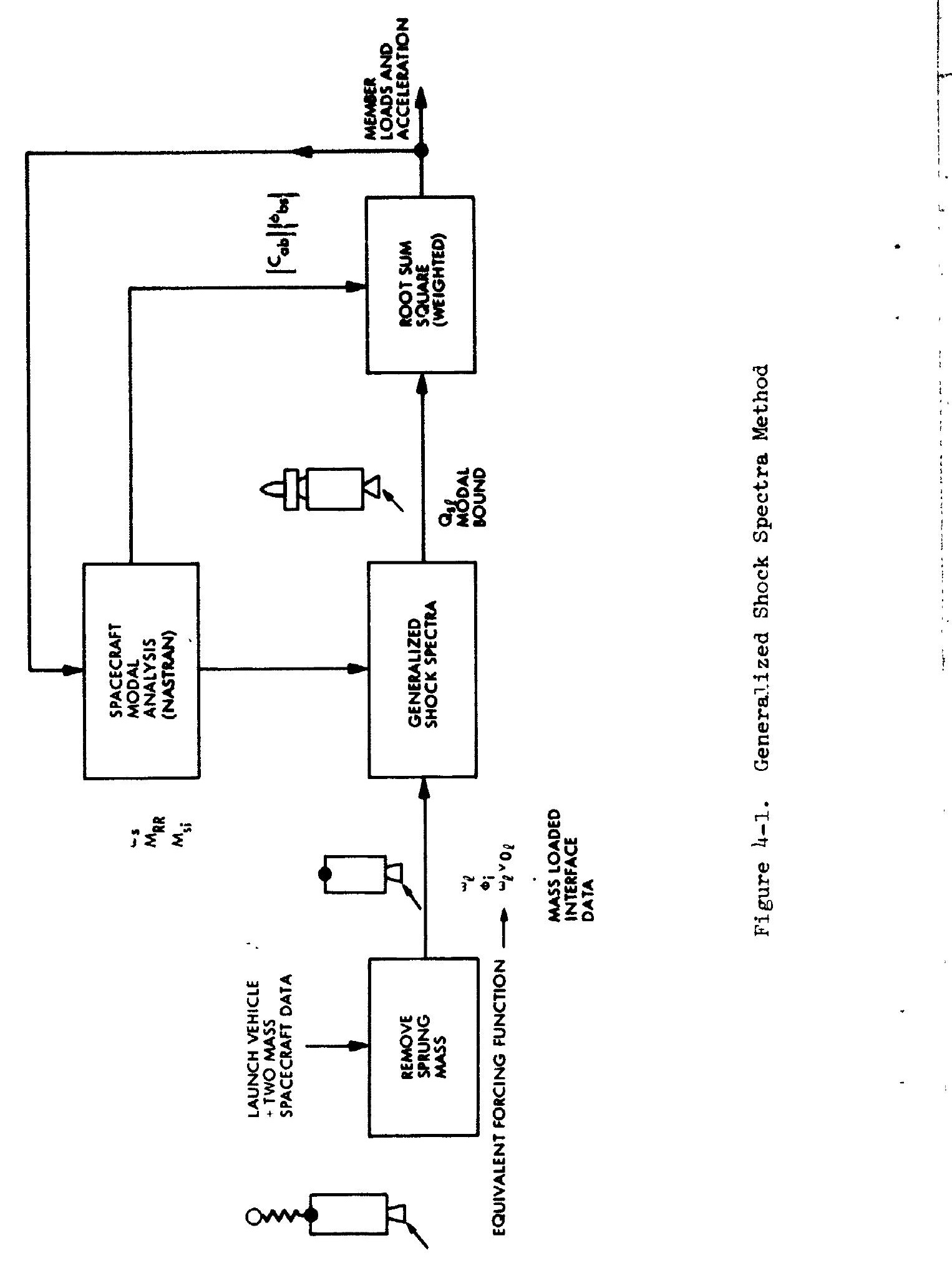




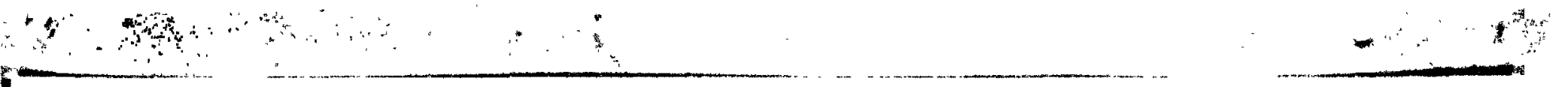

BECTION $v$

URIGDAL HAGE IS
OF POOR QUALITY

CorrCLUSIONS

The primary drawbeck of the original shock spectra method (Reference 1-1) is that it can result in very conservative answers, and therefore overdesign. It is also very dependent upon the analyst's judgment. However, it has the advantage of low cost of anaiysis and yields bounds that can be readily used for design. The transient analysis, on the other hand, is accurate, but is very expensive and difficult to implement because of its sensitivity to design change. The generalized shock spectra approach described in this paper, although still an approximate procedure, combines the advantages of low cost while maintaining a very reasonable accuracy. In addition, unlike the method of Reference 1-1, it is substantially less dependent upon the analyst's intuitive judgment in pairing spacecraft and launch vehicle modes. Because of its low cost, the tuning effect of launch vehicle modes with spacecraft mode can be very easily explored and used for design to establish worst case. Finally, the procedure is currently being applied to the Galileo spacecraft loads analysis with very acceptable results. 



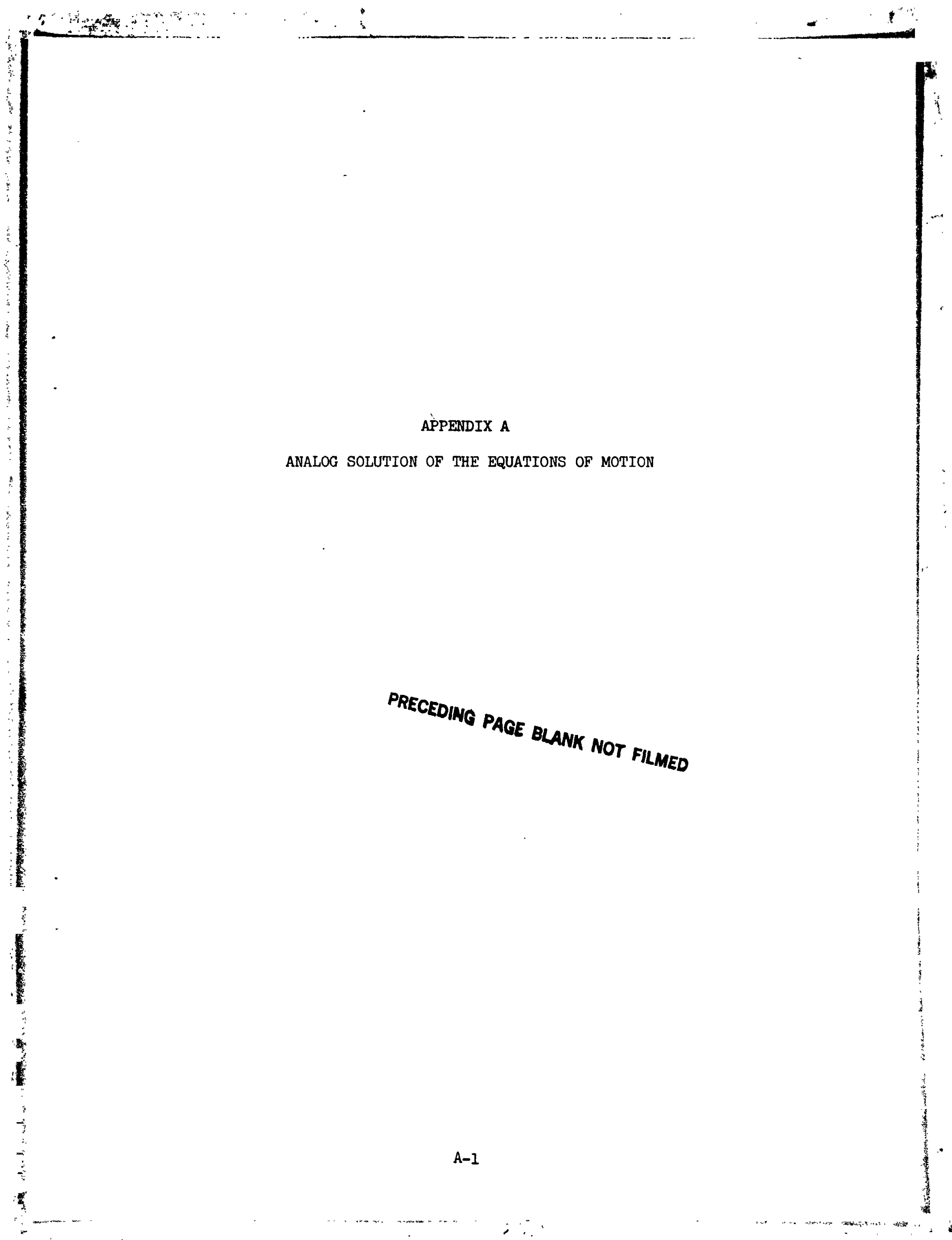


The analog exact solution of Equation (13) is given here to verify the analytical solution of Equation (15) and Appendix B. The equations of motion for the free vibration of the system in Equation (13), or that of Figure $A-1$, are

$$
\begin{array}{r}
(1+\mu) \ddot{x}_{1}+\mu \ddot{x}_{2}+2 \xi_{1} \omega_{1} \dot{x}_{1}+\omega_{1}^{2} x_{1}=0 \\
\mu \ddot{x}_{1}+\mu \ddot{x}_{2}+2 \mu \xi_{2} \omega_{2} \dot{x}_{2}+\mu \omega_{2}^{2} x_{2}=0
\end{array}
$$

where

$$
\begin{aligned}
\mu= & \text { ratio of a spacecraft mode effective mass } m_{2} \text { to a launch vehicle } \\
& \text { mode corrected effective mass } m_{1} ; \text { also, } \mu=\mu_{s l} \text { of Equation (13) } \\
\omega_{1}= & \text { corrected natural frequency of one launch vehicle mode } \\
\omega_{2}= & \text { cantilevered natural frequency of a spacecraft mode } \\
\xi_{1}= & \text { launch vehicle modal damping } \\
\xi_{2}= & \text { spacecraft modal damping } \\
x_{1}= & \text { mass } 1 \text { response (unit mass) } \\
x_{2}= & \text { mass } 2 \text { response (mass } \mu \text { ) }
\end{aligned}
$$

The solution of Equations $(A-1)$ and $(A-2)$ to an impulse force applied on mass 1 is sought. Equivalently, the initial conditions for Equations $(A-1)$ and $(A-2)$ are

$$
\begin{array}{ll}
\dot{x}_{1}(0)=F_{0} & \dot{x}_{2}(0)=0 \\
x_{1}(0)=0 & x_{2}(0)=0
\end{array}
$$

where $F_{0}$ is a constant.

To apply the initial conditions of Equation (A-3), the right hand side of Equations $(A-1)$ and $(A-2)$ must be

$$
\begin{aligned}
& F_{1}=(1+\mu) F_{0} \delta(t) \\
& F_{2}=\mu F_{0} \delta(t)
\end{aligned}
$$




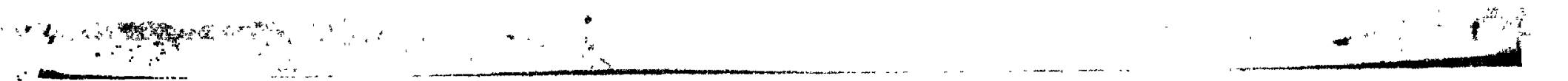

Flgure A-2 shows the anslog diagram corresponding to Equations (A-1) and $(A-2)$ solved by applying initial conditions of Equation (A-3). FiBure $A-3$ shows the analog diagram corresponding to Equations $(A-1)$ and (A-2) solved by applying the Impulse forces of Equation (A-4). These two clrcuits have been shown to give identical response time histories. All the results shown here have been obtalned using Figure A-3.

The following parametric variations were made:

(1) Frequency ratio:

$$
\frac{f_{2}}{f_{1}}=\frac{w_{2}}{w_{1}}=r=\frac{1}{R}
$$

$f_{1}$ was held at $50.00 \mathrm{~Hz}$ and $f_{2}$ was varied from $40.00 \mathrm{izz}$ to $60.00 \mathrm{~Hz}$.

(2) Dampings: $\xi_{1}=0.01,0.02,0.03$ and $\xi_{2}=0.01,0.02$, 0.03 maintaining $\xi_{1}+\xi_{2}=$ constant $=0.04$.

(3) Mass ratio: $\mu=0,0.001,0.01,0.1,1.0$.

Figure A-4 shows an example of the time histories of the sfring force per unit mass $\omega_{2}^{2} x_{2}(t)$ for mass 2 versus time for all the parameters $R, \xi_{1}, \xi_{2}$, and $\mu$. ne quantity of interest for structural loads is the largest peak value $\omega_{2}^{2} D_{\max }$ of $\omega_{2}^{2} x_{2}(t)$ of mass 2 , which is also the peak of the total acceleration $a_{m}$ of mass 2 . The peak value was read from figures similar to Figure A-4. Figure A-5 is an example of plots of the peak value $\omega_{2}^{2} D_{\max }$ versus the frequency ratio $\mathrm{R}$. The results for various values of the mass ratio $\mu$ and uanpings $\xi_{1}$ and $\xi_{2}$, keeping $\xi_{1}+\xi_{2}=0.04$ are tabulated in Table $\mathrm{A}-1$.

The solid curve on Figure A-5 has laen ubtained from a simplified Equation (23) where $\theta=0$ and where $\xi_{n}$ has been dropped in the damper nature. frequencies $\bar{\omega}_{n}$.

$$
a_{m}=\omega_{2}^{2} D_{\max }=\omega_{1} F_{0} \frac{1}{\sqrt{\Delta \Omega^{2}+4 \beta^{2}}} e^{-\alpha(2 \beta / \Delta \Omega)}
$$




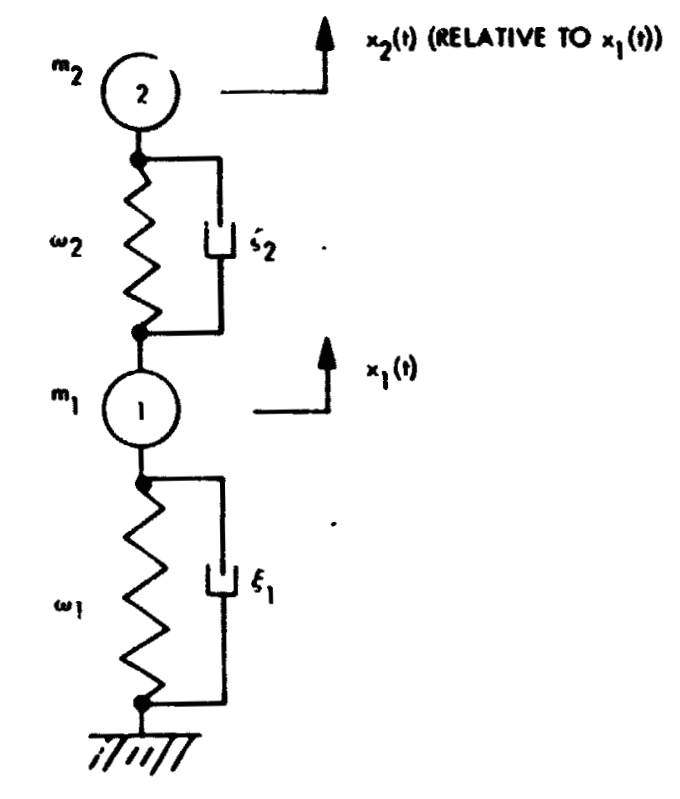

Figure A-I. Launch Vohicle - Spacecraft Modal Model

$$
\text { A-6 }
$$




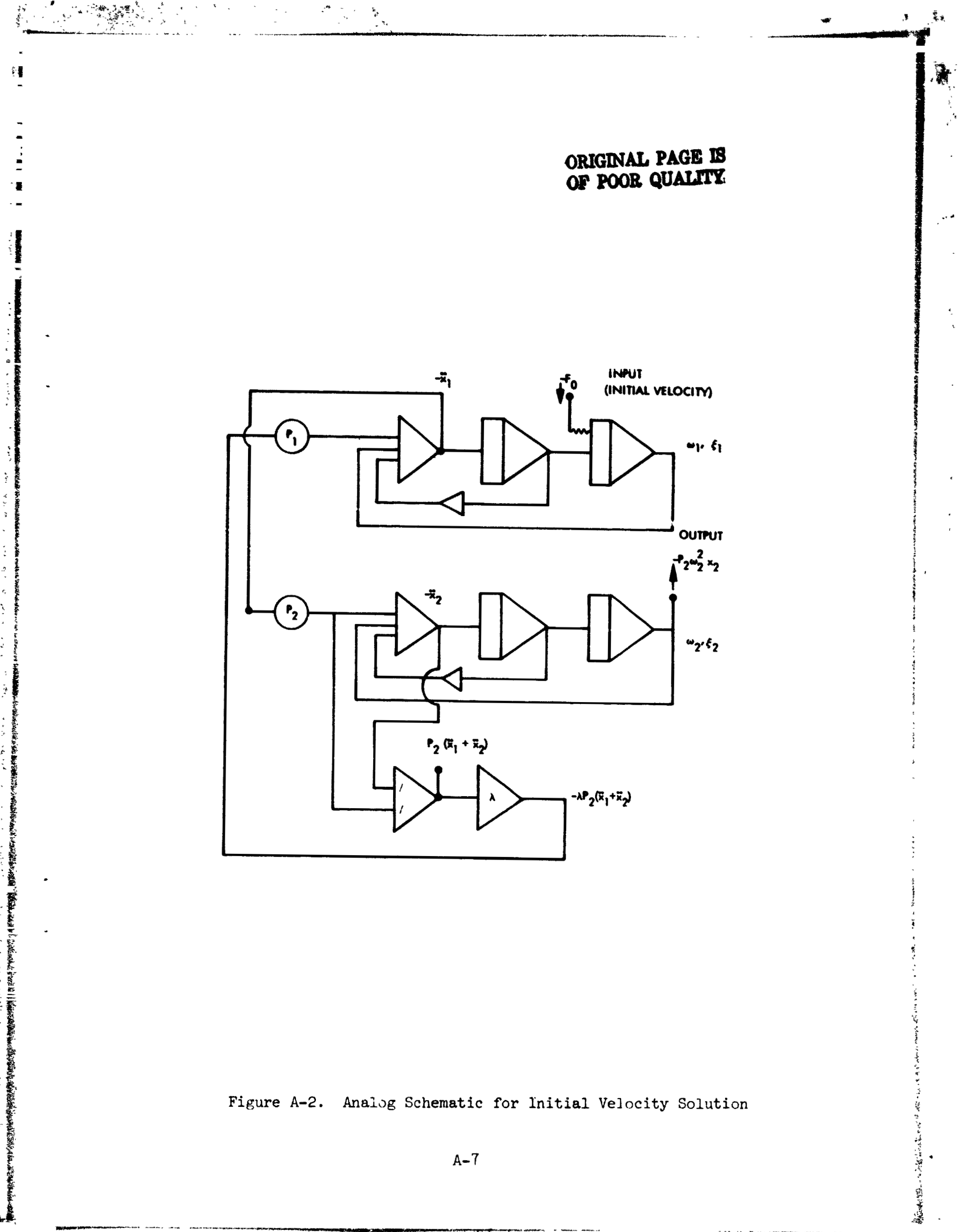




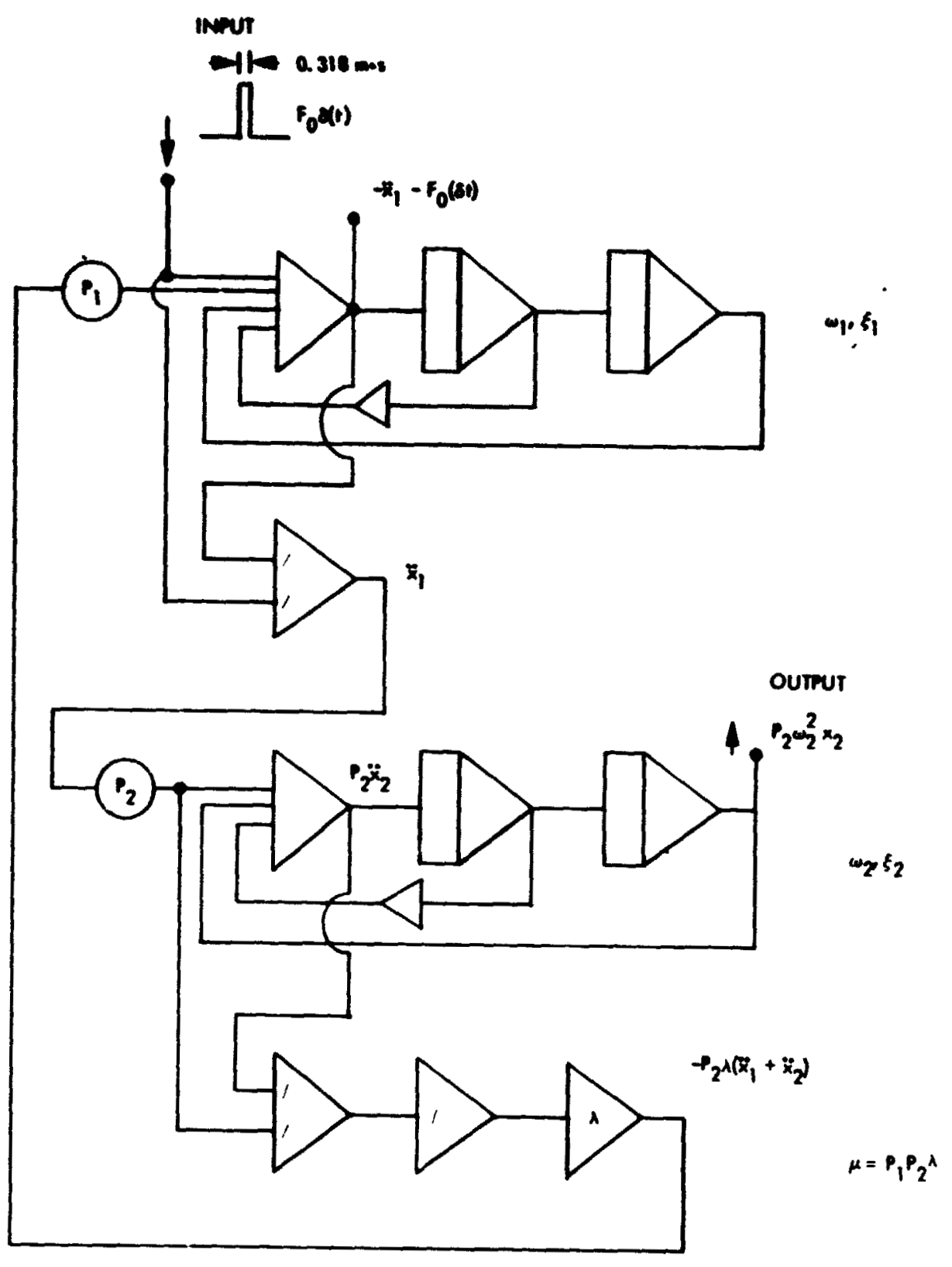

Figure A-3. Analog Schematic for Impulse solution 


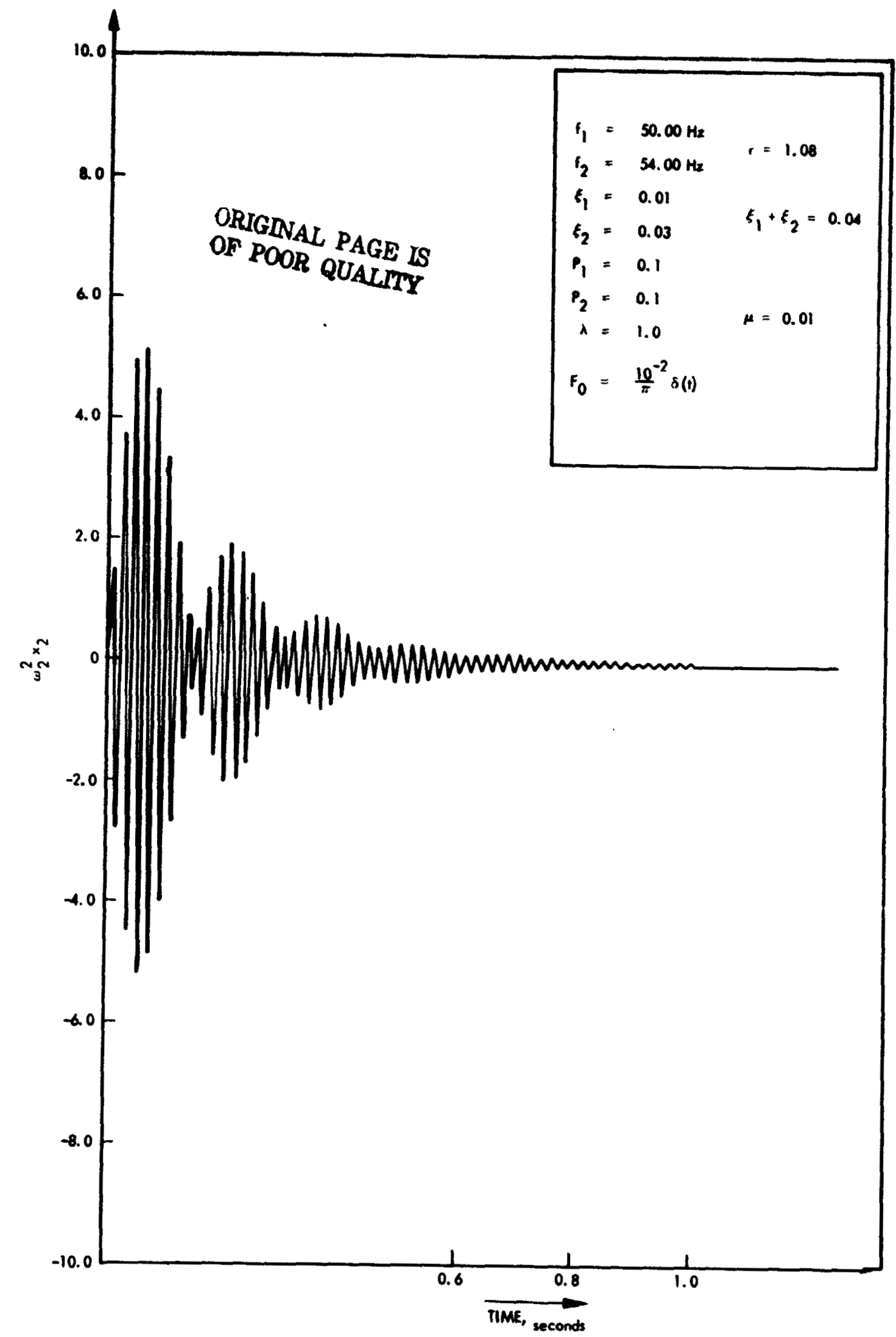

Figure A-4. Mass Number 2 Response to Unit Impulse 


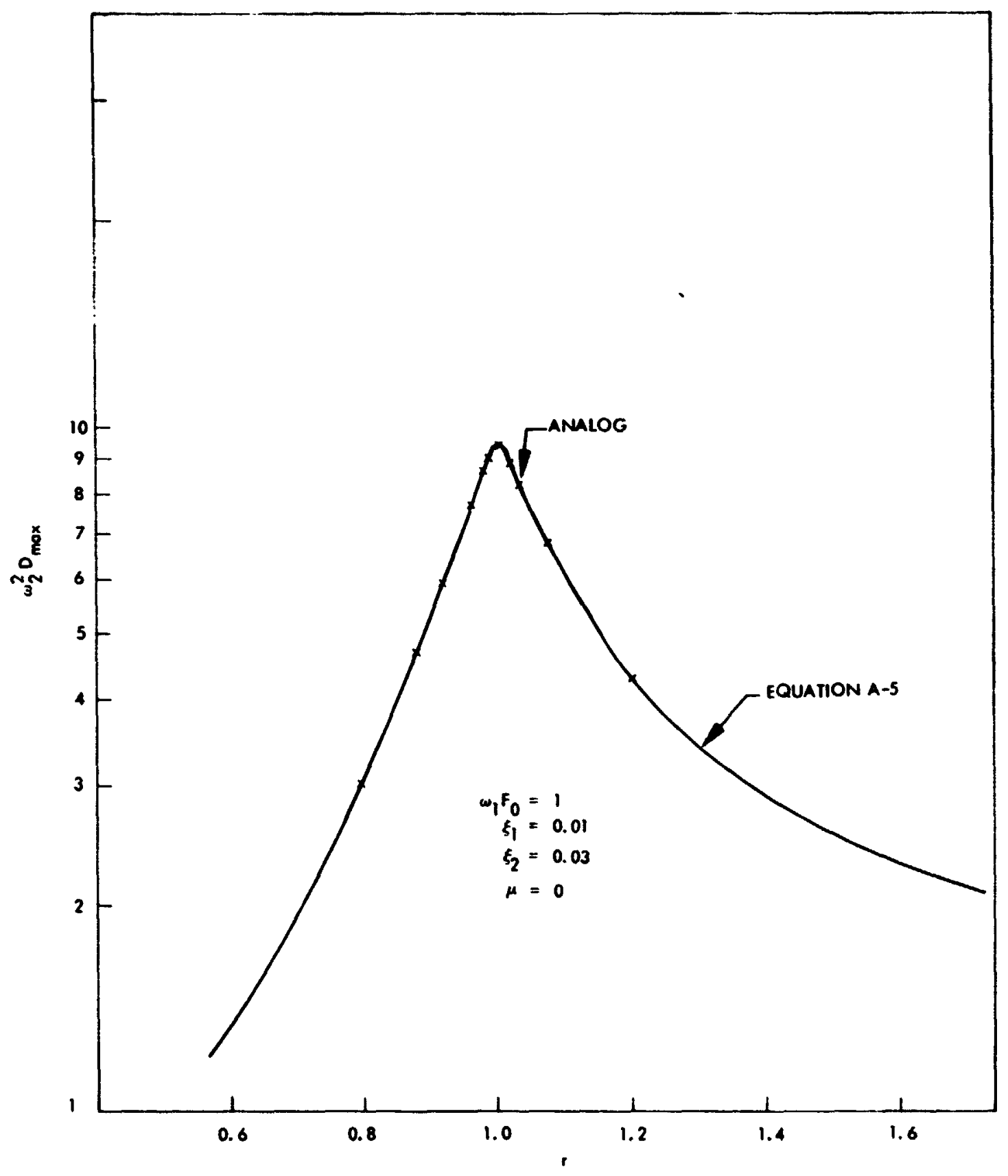

Figure A-5. Generalized Shock Spectra 


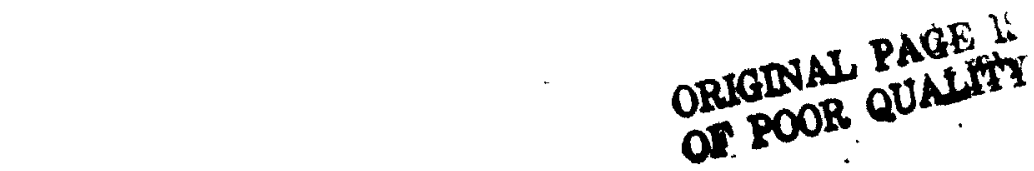

잉

"

후용

$\div$

$0 \times 4$

\section{1.}
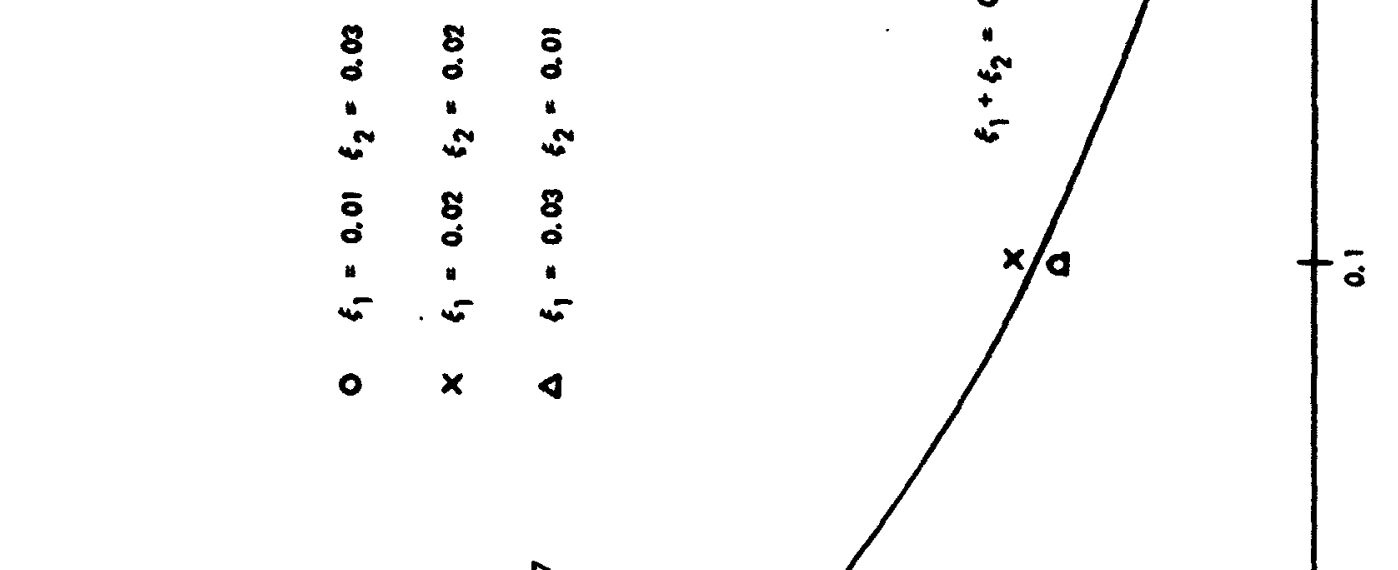

0
0
0
0
0
-1
0
0
0
0
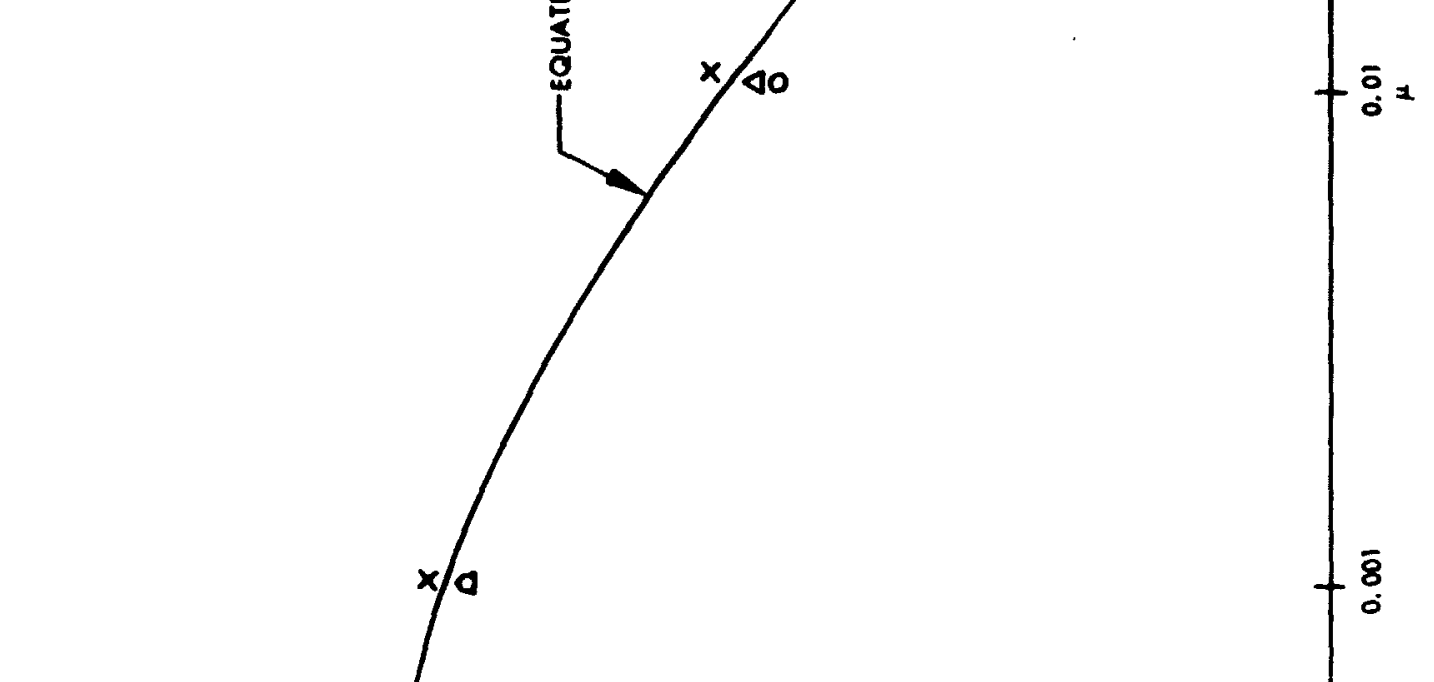


\begin{tabular}{|c|c|c|c|c|c|c|c|c|c|c|c|c|c|c|}
\hline \multirow{3}{*}{$\begin{array}{l}0 \\
\dot{-} \\
u \\
z\end{array}$} & $\begin{array}{l}m \\
0 \\
0\end{array}$ & $\begin{array}{l}\text { '0 } \\
\dot{0}\end{array}$ & & \multicolumn{11}{|c|}{$\begin{array}{l}\tilde{z} \\
\dot{0}\end{array}$} \\
\hline & ذ) & סे & & & & & & & 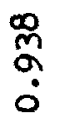 & & & & & \\
\hline & o & $\begin{array}{l}m \\
0 \\
0\end{array}$ & & & & & & & $\begin{array}{l}\stackrel{\text { nn }}{\text { ò }} \\
\dot{0}\end{array}$ & & & & & \\
\hline$\pi$ & $\stackrel{m}{\circ}$ & $\dot{0}$ & & $\begin{array}{l}\text { Oे } \\
\dot{\sim}\end{array}$ & $\begin{array}{c}\tilde{N} \\
\tilde{N}\end{array}$ & 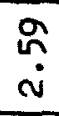 & $\begin{array}{l}\stackrel{8}{\circ} \\
\text { i }\end{array}$ & $\begin{array}{l}\vec{b} \\
\dot{v}\end{array}$ & $\begin{array}{l}8 \\
\dot{0}\end{array}$ & $\begin{array}{l}\tilde{o} \\
\stackrel{\sim}{\sim}\end{array}$ & $\begin{array}{l}8 \\
\dot{\sim}\end{array}$ & $\begin{array}{l}\vec{b} \\
\stackrel{\alpha}{0}\end{array}$ & $\begin{array}{l}\vec{n} \\
\dot{\sim}\end{array}$ & $\stackrel{n}{m}$ \\
\hline$\dot{0}$ & ه & $\begin{array}{l}\mathcal{O} \\
0\end{array}$ & $\begin{array}{l}\tilde{O} \\
\text { N }\end{array}$ & $\begin{array}{l}\stackrel{\text { mे }}{\text { c }}\end{array}$ & $\begin{array}{l}\tilde{N} \\
\tilde{N}\end{array}$ & $\begin{array}{l}\stackrel{0}{\mathscr{\sim}} \\
\dot{\sim}\end{array}$ & $\begin{array}{l}\mathscr{\infty} \\
\stackrel{\sim}{\sim}\end{array}$ & $\begin{array}{l}8 \\
\dot{\sim}\end{array}$ & $\begin{array}{l}\vec{b} \\
\dot{c}\end{array}$ & $\begin{array}{l}8 \\
\dot{\sim}\end{array}$ & $\begin{array}{l}\text { ñ } \\
\dot{i}\end{array}$ & 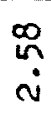 & $\begin{array}{l}m \\
\text { in }\end{array}$ & $\begin{array}{l}\vec{m} \\
\dot{\sim}\end{array}$ \\
\hline & ó & $\begin{array}{l}m \\
0 \\
0\end{array}$ & $\begin{array}{l}m \\
\text { on } \\
\text { ñ }\end{array}$ & $\begin{array}{c}\text { ले } \\
\text { i }\end{array}$ & $\overrightarrow{\tilde{n}}$ & $\begin{array}{l}\mathscr{L} \\
\dot{\sim} \\
\dot{\sim}\end{array}$ & $\begin{array}{l}\text { bे } \\
\text { i. }\end{array}$ & $\begin{array}{l}8 \\
0 \\
\text { i }\end{array}$ & $\begin{array}{l}8 \\
\stackrel{8}{c}\end{array}$ & $\begin{array}{l}\vec{b} \\
\dot{\sim}\end{array}$ & $\begin{array}{l}\vec{b} \\
\dot{v}\end{array}$ & $\begin{array}{l}8 \\
\dot{\sim} \\
\dot{0}\end{array}$ & $\begin{array}{l}\tilde{n} \\
\tilde{N}\end{array}$ & $\begin{array}{l}\vec{m} \\
\dot{\sim}\end{array}$ \\
\hline $\overrightarrow{0}$ & 웅 & $\dot{0}$ & 웅 & $\begin{array}{l}\tilde{o} \\
\dot{z}\end{array}$ & $\begin{array}{l}-1 \\
\stackrel{0}{\ddagger}\end{array}$ & $\begin{array}{l}\text { nf } \\
\text { in }\end{array}$ & $\begin{array}{l}\text { oु } \\
\text { in }\end{array}$ & $\stackrel{\stackrel{n}{ }}{\stackrel{\sim}{n}}$ & $\stackrel{\infty}{\stackrel{\infty}{\sim}}$ & $\stackrel{n}{n}$ & $\stackrel{R}{i}$ & $\stackrel{\infty}{\stackrel{\infty}{n}} \underset{\sim}{n}$ & $\begin{array}{l}\tilde{n} \\
\stackrel{n}{n}\end{array}$ & $\hat{a}$ \\
\hline$\dot{0}$ & $\begin{array}{l}\tilde{O} \\
\dot{0}\end{array}$ & $\begin{array}{l}\tilde{0} \\
\dot{0}\end{array}$ & $\begin{array}{l}\mathscr{D} \\
\text { ஸ }\end{array}$ & $\stackrel{8}{8}$ & $\stackrel{p}{?}$ & 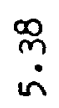 & $\begin{array}{l}8 \\
\text { in }\end{array}$ & $\begin{array}{l}\tilde{b} \\
\dot{v}\end{array}$ & $\stackrel{n}{\stackrel{n}{\sim}}$ & $\begin{array}{l}\tilde{b} \\
\text { in }\end{array}$ & $\begin{array}{l}\tilde{\sigma} \\
\dot{\sigma} \\
\dot{n}\end{array}$ & in & $\stackrel{\vec{r}}{n}$ & $\begin{array}{l}\stackrel{8}{O} \\
\dot{m}\end{array}$ \\
\hline & ó & $\begin{array}{l}\text { m. } \\
\dot{0}\end{array}$ & $\begin{array}{l}8 \\
\text { i }\end{array}$ & $\stackrel{8}{\square}$ & $\begin{array}{l}\infty \\
\stackrel{-}{+}\end{array}$ & $\begin{array}{l}\vec{J} \\
\dot{n}\end{array}$ & $\begin{array}{l}\text { மb } \\
\text { in }\end{array}$ & $\ddot{H}$ & $\begin{array}{l}\stackrel{\circ}{\infty} \\
\dot{\text { in }}\end{array}$ & $\stackrel{m}{\stackrel{m}{n}}$ & $\stackrel{P}{i}$ & $\begin{array}{l}\infty \\
\stackrel{n}{n} \\
\dot{n}\end{array}$ & $\begin{array}{l}\stackrel{n}{n} \\
\stackrel{?}{n}\end{array}$ & $\stackrel{m}{\dot{m}}$ \\
\hline$\vec{n}$ & $\stackrel{\text { m }}{\stackrel{0}{0}}$ & $\begin{array}{l}0 \\
0\end{array}$ & & $\stackrel{8}{8}$ & $\begin{array}{l}\stackrel{\sim}{\infty} \\
\stackrel{0}{n}\end{array}$ & $\begin{array}{l}\overrightarrow{\vec{J}} \\
\stackrel{0}{\sim}\end{array}$ & 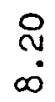 & $\stackrel{\infty}{m}$ & $\begin{array}{l}\approx \\
\approx \\
\infty\end{array}$ & $\underset{\underset{\infty}{\infty}}{\stackrel{N}{ \pm}}$ & $\stackrel{m}{m}$ & 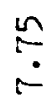 & $\begin{array}{l}\vec{\omega} \\
\dot{\omega}\end{array}$ & $\underset{\widetilde{N}}{\tilde{J}}$ \\
\hline $\begin{array}{l}0 \\
\dot{0} \\
\text { " }\end{array}$ & $\begin{array}{l}\tilde{O} \\
\dot{0}\end{array}$ & $\begin{array}{l}\tilde{0} \\
\dot{0}\end{array}$ & $\begin{array}{l}\text { o } \\
\dot{m}\end{array}$ & $\stackrel{n}{n}$ & $\stackrel{n}{\curvearrowleft}$ & 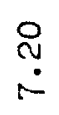 & 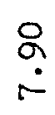 & $\begin{array}{l}\vec{W} \\
\dot{\infty} \\
\dot{0}\end{array}$ & 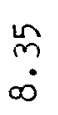 & $\stackrel{p}{m}$ & $\begin{array}{l}\% \\
\infty\end{array}$ & $\stackrel{\infty}{\stackrel{n}{n}}$ & $\begin{array}{l}\vec{z} \\
\dot{0}\end{array}$ & 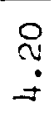 \\
\hline & $\begin{array}{l}0 \\
\dot{0}\end{array}$ & $\begin{array}{l}m \\
0 \\
0\end{array}$ & & $\stackrel{n}{n} \stackrel{n}{\tilde{z}}$ & $\begin{array}{l}\infty \\
\stackrel{\infty}{1}\end{array}$ & $\stackrel{\stackrel{m}{\mathrm{r}}}{\stackrel{5}{\circ}}$ & 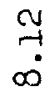 & $\vec{m}$ & $\stackrel{n}{\sim} \stackrel{n}{\infty}$ & $\underset{\infty}{\stackrel{\sim}{*}}$ & $\stackrel{p}{m}$ & $\stackrel{\infty}{\stackrel{\infty}{\leftarrow}}$ & $\tilde{i n}$ & $\underset{\overrightarrow{\mathrm{v}}}{\overrightarrow{\mathrm{z}}}$ \\
\hline & $\stackrel{m}{c}$ & $\overrightarrow{0}$ & $\begin{array}{l}\stackrel{2}{0} \\
\dot{m}\end{array}$ & $\stackrel{?}{\because}$ & $\begin{array}{l}\circ \\
\circ \\
\text { : }\end{array}$ & $\begin{array}{l}\stackrel{L}{\infty} \\
\stackrel{0}{\leftarrow}\end{array}$ & $\begin{array}{l}\stackrel{\alpha}{\infty} \\
\dot{\infty}\end{array}$ & $\begin{array}{l}\infty \\
\stackrel{-}{a} \\
0\end{array}$ & $\begin{array}{l}\stackrel{n}{m} \\
\dot{\sigma}\end{array}$ & $\begin{array}{l}\stackrel{n}{a} \\
\stackrel{a}{a}\end{array}$ & $\begin{array}{l}0 \\
\dot{0} \\
0\end{array}$ & $\stackrel{n}{\stackrel{n}{\infty}}$ & $\begin{array}{l}8 \\
\infty \\
\dot{6}\end{array}$ & $\stackrel{m}{m}$ \\
\hline " & $\begin{array}{l}\tilde{0} \\
\dot{0}\end{array}$ & $\begin{array}{l}\mathscr{\theta} \\
\dot{0}\end{array}$ & 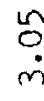 & $\begin{array}{l}\text { ?n } \\
=\end{array}$ & $\begin{array}{l}\vec{a} \\
\dot{\sim}\end{array}$ & 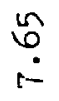 & $\stackrel{\sim}{\sim}$ & $\begin{array}{l}8 \\
\infty \\
\infty\end{array}$ & $\begin{array}{l}8 \\
\dot{a}\end{array}$ & $\begin{array}{l}\mathscr{\infty} \\
\infty \\
\infty\end{array}$ & $\begin{array}{l}\mathscr{0} \\
\infty \\
\infty\end{array}$ & $\begin{array}{l}g \\
\infty\end{array}$ & $\begin{array}{l}\overline{6} \\
\dot{6}\end{array}$ & $\begin{array}{l}\stackrel{P}{?} \\
\stackrel{7}{*}\end{array}$ \\
\hline & $\overrightarrow{0}$ & $\begin{array}{l}\text { m } \\
\dot{0}\end{array}$ & $\begin{array}{l}\stackrel{\leftrightarrow}{0} \\
\dot{m}\end{array}$ & : & $\stackrel{8}{8}$ & $\begin{array}{l}\infty \\
\stackrel{0}{-0}\end{array}$ & $\stackrel{8}{\infty}$ & $\stackrel{m}{\vec{b}}$ & $\begin{array}{l}m \\
\stackrel{-}{a} \\
\dot{a}\end{array}$ & $\stackrel{\check{\sigma}}{\check{\sigma}}$ & $\begin{array}{l}\vec{a} \\
\dot{a}\end{array}$ & $\stackrel{m}{\infty}$ & $\begin{array}{l}0 \\
0 \\
\dot{0}\end{array}$ & $\dot{g}$ \\
\hline & & & $\begin{array}{l}\infty \\
0 \\
0\end{array}$ & $\begin{array}{l}\infty \\
\infty \\
\dot{0}\end{array}$ & $\stackrel{g}{0}$ & $\begin{array}{l}0 \\
\circ \\
0\end{array}$ & 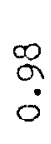 & $\stackrel{g}{\circ}$ & : & $\stackrel{-1}{0}$ & $\underset{\tilde{y}}{\tilde{y}}$ & ن. & $\underset{\dot{r}}{\infty}$ & $\stackrel{\ominus}{\dddot{-1}}$ \\
\hline
\end{tabular}


ORIGLAL PAGE IS

OF POOR QUALIII

\section{Eiscroung PACE BunK not FIMED}

APPDANTX B

ANALYTICAL SOLUTION OF THE EQUATIONS OF MOTION 
Analytical solution is derived here in detall for the coupled equation of motion (13):

$$
\begin{aligned}
{\left[\begin{array}{l|l}
(1+\mu) & \mu \\
\hline \mu & \mu
\end{array}\right]\left\{\begin{array}{c}
\ddot{x}_{1} \\
\ddot{x}_{2}
\end{array}\right\} } & +\left[\begin{array}{c|c}
2 \xi_{1} \omega_{1} & 0 \\
\hline 0 & 2 \xi_{2} \omega_{2} \mu
\end{array}\right]\left\{\begin{array}{l}
\dot{x}_{1} \\
\dot{x}_{2}
\end{array}\right\} \\
& +\left[\begin{array}{c|c}
\omega_{1}^{2} & 0 \\
\hline 0 & \omega_{2}^{2} \mu
\end{array}\right]\left\{\begin{array}{c}
x_{1} \\
x_{2}
\end{array}\right\}=\left\{\begin{array}{c}
F_{0 \ell}^{(t)} \\
0
\end{array}\right\}
\end{aligned}
$$

An analng solution to Equation ( $B-1$ ) above has oeen given in Appendix $A$. The general solution takes a complex form, with real and imaginary parts. One approach to obtaining the analytical solution to Equation $(B-1)$ is by first deriving and using the modal coordinates of the undamped system in Equation (B-I) to diagonalize the mass and stiffness matrices. This, however, will not diagonalize the damping matrix, but the common approximation of leaving out the off-diagonal terms will result in retaining only the real part of the solution. This is an acceptable approximation for small damping.

Let us first write the frequency equation for the damped system since properties of the damped frequency equation will be used. The form of the solution is

$$
\left.\left[\begin{array}{l}
x_{1} \\
x_{2}
\end{array}\right\}=e^{p t} \quad \begin{array}{l}
y_{1} \\
y_{2}
\end{array}\right\}
$$

Substituting in the left-hand side of Equation ( $B-1$ ) results in the damped frequency equation:

$$
\begin{gathered}
\left(\frac{p}{\omega_{2}}\right)^{4}+2\left[R \xi_{1}+(1+\mu) \xi_{2}\right]\left(\frac{p}{\omega_{2}}\right)^{3}+\left[R^{2}+4 R \xi_{1} \xi_{2}+(1+\mu)\right]\left(\frac{p}{\omega_{2}}\right)^{2} \\
+2 R\left[\xi_{1}+\xi_{2} R\right]\left(\frac{p}{\omega_{2}}\right)+R^{2}=0
\end{gathered}
$$


The undamped frequency equation is then obtained by letting

$$
\xi_{1}=\xi_{2}=0
$$

$$
\Omega^{4}-\left[R^{2}+(1+\mu)\right] \Omega^{2}+R^{2}=0
$$

where

$$
\begin{aligned}
& \Omega=\frac{\omega}{\omega_{2}} \\
& R=\frac{\omega_{1}}{\omega_{2}}=\frac{\omega_{l}}{\omega_{s} \sqrt{1+M_{s l}}}
\end{aligned}
$$

The roots to Equation $(B-3)$ are $\omega_{n 1}=\Omega_{1} \omega_{2}$ and $\omega_{n 2}=\Omega_{2} \omega_{2}$ in which $\Omega_{1}$ and $\Omega_{2}$ are defined by

$$
\begin{aligned}
& \Omega_{1}=\Omega_{0}-\frac{\Delta \Omega}{2} \\
& \Omega_{2}=\Omega_{0}+\frac{\Delta \Omega}{2}
\end{aligned}
$$

and $\Omega_{0} \omega_{2}$ and $\Delta \Omega_{2} \omega_{2}$, respectively, are the center and the beat frequency:

$$
\begin{aligned}
& \omega_{2} \Omega_{0}=\frac{\omega_{2}}{2} \sqrt{(R+1)^{2}+\mu} \\
& \omega_{2} \Delta \Omega=\omega_{2} \sqrt{(R-1)^{2}+\mu}
\end{aligned}
$$

Associated with the frequencies $\omega_{n 1}$ and $\omega_{n 2}$ obtained above are two undamped mode shapes, respectively defined by 


$$
\left\{Y_{1}\right\}=\left\{\begin{array}{c}
\frac{1}{\Omega_{1}^{2}}-1 \\
-\cdots \\
1
\end{array}\right\} \text { and }\left\{Y_{2}\right\}=\left\{\begin{array}{c}
\frac{1}{\Omega_{2}^{2}}-1 \\
-2-\ldots \\
1
\end{array}\right\}
$$

Next, the classical coordinate transformation is applied to $x_{1}$ and $x_{2}$ to diagonalize the matrices of Equation $(B-1)$ :

$$
\left(\begin{array}{l}
x_{1} \\
x_{2}
\end{array}\right\}=\left[y_{1} \mid y_{2}\right]\left\{\begin{array}{l}
z_{1} \\
z_{2}
\end{array}\right\}
$$

This results in:

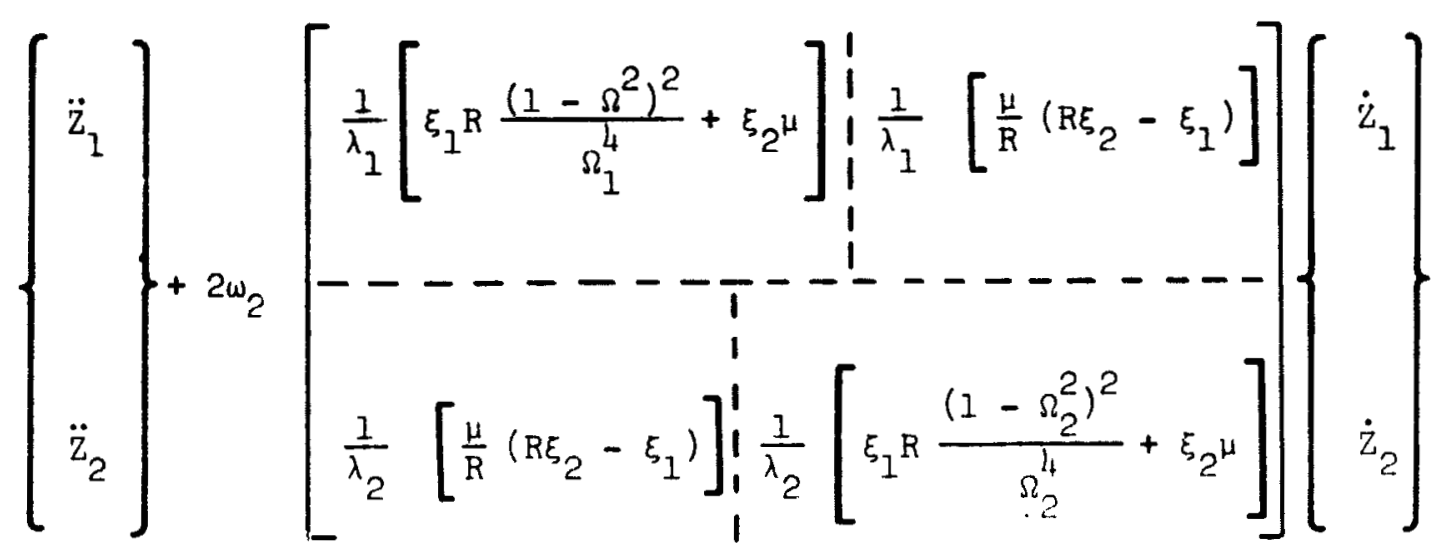

where

$$
+\left[\begin{array}{c|c}
\omega_{n 1}^{2} & 0 \\
\hline 0 & \omega_{n 2}^{2}
\end{array}\right]\left\{\begin{array}{l}
z_{1} \\
z_{2}
\end{array}\right\}=\left\{\begin{array}{l}
\frac{1}{\lambda_{1}}\left(\frac{1}{\Omega_{1}^{2}}-1\right) \\
\frac{1}{\lambda_{2}}\left(\frac{1}{s_{2}^{2}}-1\right)
\end{array}\right\} F_{0}(t)
$$

$$
\begin{aligned}
& \lambda_{1}=\frac{1}{\Omega_{1}^{4}}\left[\left(1-s_{1}^{2}\right)^{2}+\mu\right] \\
& \lambda_{2}=\frac{1}{\Omega_{2}^{4}}\left[\left(1-\Omega_{2}^{2}\right)^{2}+\mu\right]
\end{aligned}
$$

are the generalized mass. 


\section{ORIGNAL PÄGE IS \\ OF POOR QUALTIY}

It is ceen that the off-diagonal terms in the damping matrix will vanish when the demping ratio is proportional to the frequency ratio. No such limitation will be imposed, and therefore, $\xi_{1} / \xi_{2} \neq \omega_{1} / \omega_{2}$. However, the magnitude of the off-diagonal terms will be assumed small enough to be neglected in subsequent calculations. This is equivalent to neglecting the imaginary part of the solution, a commonily used approximation that results in decoupling Equation (B-9), so that

$$
\begin{aligned}
& \ddot{z}_{1}+2 \xi_{n 1} \omega_{n 1} \dot{z}_{1}+\omega_{n 1}^{2} z_{1}=\frac{1-\Omega_{1}^{2}}{\Omega_{1}^{2} \lambda} F_{0 l}(t) \\
& \ddot{z}_{2}+2 \xi_{n 2} \omega_{n 2} \dot{z}_{2}+\omega_{n 2}^{2} z_{2}=\frac{1-\Omega_{2}^{2}}{\Omega_{2}^{2} \lambda_{2}} F_{0 l}(t)
\end{aligned}
$$

where now:

$$
\begin{aligned}
& 2 \omega_{n 1} \xi_{n 1}=\frac{\alpha_{2}}{\lambda_{1}}\left[\xi_{1} R \frac{\left(1-\Omega_{1}^{2}\right)^{2}}{\Omega_{1}^{4}}+\xi_{2}{ }^{\mu}\right] \\
& 2 \omega_{n 2^{\xi} \xi_{2}}=\frac{2 \omega_{2}}{\lambda_{2}}\left[\xi_{1} R \frac{\left(1-\Omega_{2}^{2}\right)^{2}}{\Omega_{2}^{4}}+\xi_{2}^{\mu}\right]
\end{aligned}
$$

Furthermore, from the theory of equation, the sum of the roots of the damped frequency equation, Equation $(B-3)$, is equel to the coefficient of $\left(p / w_{2}\right)^{3}$. Therefore:

$$
\xi_{n 1} \omega_{n 1}+\xi_{n 2} \omega_{n 2}=2 \beta \omega_{2}
$$

where

$$
B=\frac{1}{2}\left[\xi_{j} R+(1+\mu) \xi_{2}\right]
$$


The following approximate relationship will be useful:

$$
\xi_{n 1} \omega_{n 1}-\xi_{n 2} \omega_{n 2}=2 \theta \omega_{2}
$$

where

$$
\theta=\frac{1}{\lambda_{1}}\left[\xi_{1} R\left(\frac{1}{\Omega_{1}^{2}}-1\right)^{2}+\xi_{2} \mu\right]-\frac{1}{2}\left[\xi_{1} R+(1+\mu) \xi_{2}\right]
$$

Equations (B-10) may now be solved for $E$ prescribed force system $F_{O \ell}(t)$. The specific case when $F_{\partial \ell}(t)$ is an inpulse delta function of amplitude $F_{0 l}$ applied at $t=0$ is discussed. Fy definition, the impulse $F_{0 \ell}(t)=F_{O \ell} \delta(t)$ is the response of an initial velocity $v_{O l}$. Tumerically $F_{O l}=v_{O l}$ for an impulse applied to a unit mass, as it is the case for Equation (B-9). The homogeneous solution of Equation (B-10) is:

$$
z_{r}=A_{r} e^{-\xi n r^{\omega} n r^{t}} \sin \bar{\omega}_{n r} t+B_{r} e^{-\xi} n r^{\omega} n r^{t} \cos \bar{\omega}_{11 r} t
$$

where $\bar{\omega}_{n r}=\omega_{n r} \sqrt{1-\xi_{n r}^{2}}=$ damped natural frequency and $A_{r}, B_{r}$ are constants ( $r=1$ for launch vehicle; $r=2$ for the spacecraft).

The initial conditions for the system at $t=0$ are

$$
\begin{aligned}
& x_{1}(0)=x_{2}(0)=0 \\
& \dot{x}_{1}(0)=v_{0 l} \text { and } \dot{x}_{2}(0)=0
\end{aligned}
$$

By combining the transformation of $(B-8)$ with the general solution of $(B-13)$ and the initial conditions in (B-14), we have 


\section{URIGNAL PAGE IS \\ OF POOR QUALTYY}

$$
\begin{aligned}
\lambda_{1}(t)= & \frac{v_{0 l}}{\left(\Omega_{2}^{2}-\Omega_{1}^{2}\right)}\left[\frac{\Omega_{2}^{2}\left(1-\Omega_{1}^{2}\right)}{\bar{\omega}_{n 1}} e^{-\xi_{n 1} \omega_{n 1} t} \sin \bar{\omega}_{n 1} t\right. \\
& \left.-\frac{\Omega_{1}^{2}\left(1-\Omega_{2}^{2}\right)}{\bar{\omega}_{n 2}} c^{-\xi_{n 2} \omega_{n 2} t} \sin \bar{\omega}_{n 2} t\right] \\
x_{2}(t)= & \frac{R^{2} v_{0 l}}{\left(\Omega_{2}^{2}-\Omega_{1}^{2}\right)}\left[\frac{1}{\bar{\omega}_{n 1}} e^{-\bar{t}_{n 1} \omega_{n 1} t} \sin \bar{\omega}_{n 1} t\right. \\
& \left.-\frac{1}{\bar{\omega}_{n 2}} e^{-\xi_{n 2}{ }_{n 2} t} \sin \bar{\omega}_{n 2} t\right]
\end{aligned}
$$




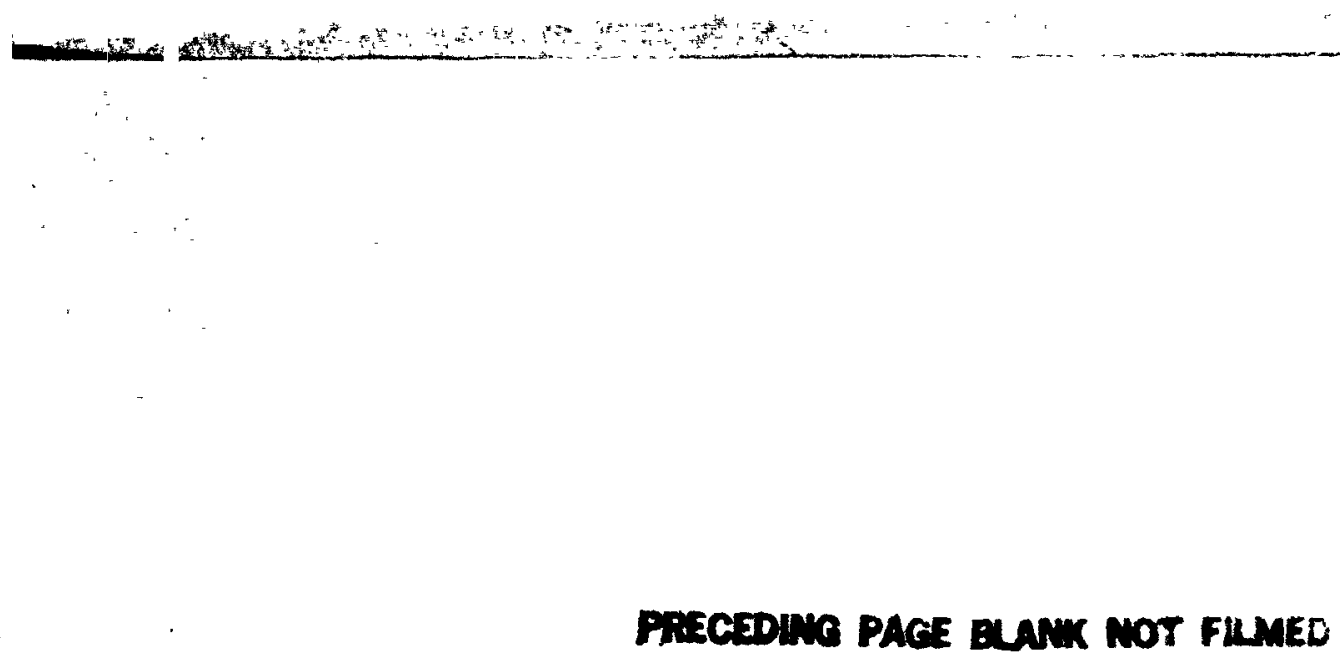

APPENDIX C

RECOVERY OF LAUNCH VEHICLE MODAL PROPERTIES

Cl 


\section{A. EQUATIONS OF MOTION}

In this appendix, equations are derived whereby the modes and modal responses of the launch vehicle with an interface loaded by only a rigid mass can be recovered from the given modes and modal responses of the same launch vehicle whose interface is loaded by an elastic and a rigid-mass simulation of the spacecraft. Let the subscripts $S$ denote cantilevered spacecraft modes, and the subscripts $C$ denote the composite launch vehicle free-free modes with an elastically loaded interface, see Figure C-1. The motion of the total composite system of spacecraft and launch vehicle in Figure $C-1$ subjected to an external force $\left\{F_{j}\right\}$ is governed by the set of equations:

$$
\left\{\bar{q}_{c}\right\}+\left[2 \xi_{c} \omega_{c}\right]\left\{\dot{q}_{c}\right\}+\left[\omega_{c}^{2}\right]\left(q_{c}\right\}=\left[\phi_{c j}\right]\left(F_{j}\right\}
$$

where $\ddot{q}_{c}$ are available from the launch vehicle analysis.

The modes of the launch vehicle with a rigid-mass can be computed from Equation $(\mathrm{C}-1)$ by subtracting the modal reactions of the elastic spacesraft at the interface. This is analogous to Equation (2) of the main text in which the sign of the spacecraft reaction force $\left\{F_{i}\right\}$ is negative since the spacecraft that was originally put on the launch vehicle is now being removed. In this case, the feneralized coordinates are denoted by $\left\{q_{c}\right\}$ insteud of $\left\{q_{c}\right\}$ :

$$
\left\{\ddot{q}_{c}^{\prime}\right\}+\left[2 \xi_{c} \omega_{c}\right]\left\{\dot{q}_{c}^{\prime}\right\}+\left[\omega_{c}^{2}\right]\left\{q_{c}^{\prime}\right\}=\left[\phi_{c j}\right]\left\{F_{j}\right\}-\left[\phi_{c i}\right]\left\{-F_{i}\right\}
$$

From Equations $(8)$ and $(4)$ :

$$
\begin{aligned}
& \left\{F_{i}\right\}=\left[M_{i i}\right]\left\{\ddot{k}_{i}\right\}+\left[m_{i s}\right]\left\{\ddot{q}_{s}^{\prime}\right\} \\
& \left\{\ddot{R}_{i}\right\}=[\phi .]\left\{\ddot{q}_{c}^{\prime}\right\} \\
& {\left[M_{i i}\right]=\left[M_{i j}\right]_{R E S}+\sum_{s=1}^{S}\left[m_{i i}^{s}\right]}
\end{aligned}
$$




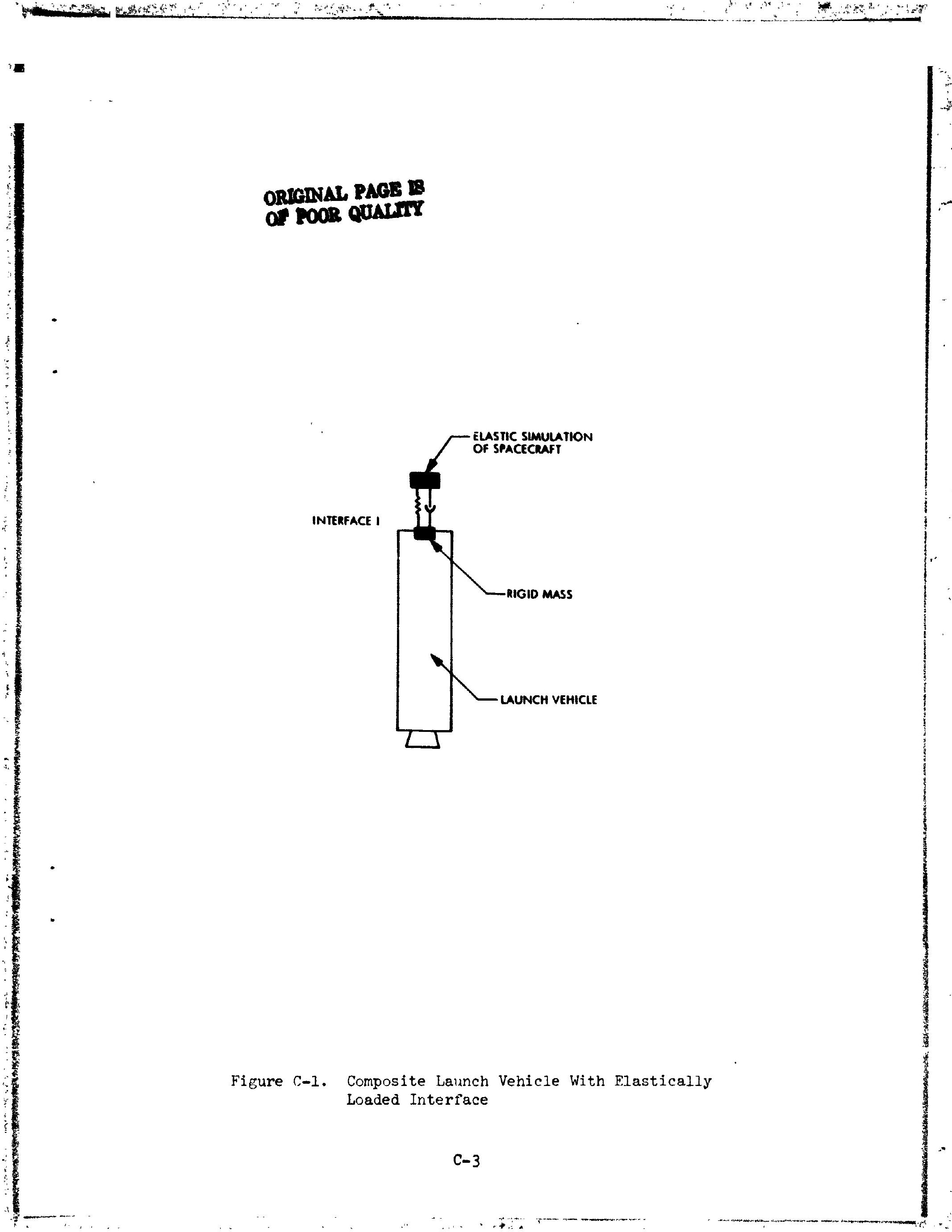


Also, from Equation (3) of the main text, the spacecraft is governed by:

$$
\left\{\ddot{q}_{s}^{\prime}\right]+\left[2 \xi_{s} \omega_{s}\right]\left\{\ddot{q}_{s}^{\prime}\right\}+\left[\omega_{s}^{2}\right]\left(q_{s}^{\prime}\right\}=-\left[m_{s i}\right]\left[\ddot{R}_{i}\right\}
$$

Equations $(\mathrm{c}-2, \mathrm{C}-3$, and $\mathrm{c}-4)$ are now combined to give th zuverning eruations for the rigid-mass-loaded leunch velifile response:

$$
\begin{aligned}
& {\left[\begin{array}{c|c}
(I I]-\left[\phi_{c i}\right]\left[M_{i i}\right]\left[\phi_{i c} l\right) & -\left[\phi_{c i}\right]\left[m_{i s}\right] \\
\hline-\left[m_{s i}\right]\left[\phi_{i r}\right] & -[I]
\end{array}\right]\left\{\begin{array}{c}
\ddot{q}_{c}^{\prime} \\
\ddot{q}_{s}^{\prime}
\end{array}\right\}} \\
& +\left[\begin{array}{c|c}
2 \xi_{c} \omega_{c} & 0 \\
\hline 0 & -2 \xi_{s} \omega_{s}
\end{array}\right]\left\{\begin{array}{c}
\dot{q}_{c}^{\prime} \\
\dot{q}^{\prime}
\end{array}\right\}+\left[\begin{array}{c|c}
\omega_{c}^{2} & 0 \\
\hline 0 & -\omega_{s}^{2}
\end{array}\right]\left\{\begin{array}{c}
q_{c}^{\prime} \\
q_{s}^{\prime}
\end{array}\right\}=\left\{\begin{array}{c}
{\left[\phi_{c j}\right]\left(F_{j}\right\}} \\
-\frac{1}{0}
\end{array}\right\}(c-5)
\end{aligned}
$$

In a concise form, Equation $(C-5)$ is written as:

$$
[\tilde{M}]\left\{\vec{q}^{\prime}\right\}+[\tilde{D}]\left\{\dot{q}^{\prime}\right\}+\left[\bar{\omega}^{2}\right]\left\{q^{\prime}\right\}=\{F\}
$$

and its undamped homogeneous part is written as

$$
[\tilde{\mathrm{M}}]\left[\ddot{q^{\prime}}\right\}+\left[\tilde{\omega}^{2}\right]\left\{\dot{q}^{\prime}\right\}=0
$$

\section{B. EIGENVALUE SOLUTION}

In general, if the system of composite modes, $C$, conta+ised S-spacecraft elastic modes, and L-launch vehicle rigid-and-ela itic modes $C=S+L$, the undamped eigenvalue solution of Equation $(C-6)$ will result in $(L+2 S)$ eigenvalues $\Omega_{L}, \Omega_{S 1}, \Omega_{s 2}$ and associated eigenvectors $\left\{\mathrm{v}_{\mathrm{L}}\right\},\left\{\mathrm{V}_{\mathrm{s} 1}\right\},\left\{\mathrm{V}_{\mathrm{s} 2}\right\}$.

of these, two S-roots will be repeated, $\Omega_{s 1}=\Omega_{s 2}$, and are associated with the elastic modes of the spacecraft. The remaining L-roots are associated with the rigid-mass-loaded launch vehicle free-free modes, 
some of which will correspond to rigtd body modes with sero frequencies, and the remaining modes will correspond to elastic modes with nonsero rrequencies. The $(L+2 S)$ set of modes collectively referred to as [V]

$$
[v]=\left[v_{L}\left|v_{s 1}\right| v_{s 2}\right]
$$

will transform Equation $(c-6)$ to the diagonal form

$$
[I][Q]+\left[\Omega^{2}\right](Q)=0
$$

with

$$
\left\{q^{\prime}\right\}=[v]\{Q\}
$$

C. MODE SHAPES AND ACCELERATIONS AT THE INTERFACE

The acceleration $\left(\ddot{R}_{i}\right)$ at the interface can be expressed in either the $\left\{q^{\prime}\right\}$ or $\{Q\}$ coordinates. Thus,

$$
\left[\ddot{R}_{i}\right\}=\left[\phi_{i}\right]\left[\ddot{q}^{\prime}\right\}
$$

or

$$
\left\{\ddot{R}_{i}\right\}=\left[r_{i}\right][\ddot{Q}\}
$$

where $\left[\phi_{i}\right]$ and $\left[\psi_{i}\right]$ are the mode shapes at the interface in the $\left\{q^{\prime}\right\}$ and the $\{G\}$ coordinates, respectively.

Because of Equation (c-9),

$$
\begin{aligned}
\left\{\ddot{R}_{i}\right\} & =\left[\Phi_{i}\right]\left[\ddot{q} \dot{q}^{\prime}\right\}=\left[\phi_{i}\right][v][\ddot{Q}\} \\
& =\left[\psi_{i}\right]\{\ddot{Q}\}
\end{aligned}
$$

then

$$
\left[\psi_{i}\right]=\left[\phi_{i}\right][v]
$$




\section{MODAL ACCELERATION TIME RISTCRY $\{\ddot{Q}(t)\}$}

The time history of the modal acceleration corresponding to the newly obtained rigid-mass-loaded launch vehicle modes $\{Q\}$ are obtained from Equation (c-9) so that

$$
[\ddot{Q}]=\left[v^{-1}\right][\ddot{q}]
$$

The inverse $\left[\mathrm{V}^{-1}\right]$ in Equation $(\mathrm{C}-12)$ need not be performed. It can be found from the orthogonality condition that led to Equation $(c-8)$ :

$$
\left[v^{T}\right][\tilde{M}][v]=[I]
$$

so that

$$
\left[\mathrm{v}^{-1}\right]=\left[\mathrm{v}^{\mathrm{T}}\right][\tilde{\mathrm{M}}]
$$

Therefore,

$$
[\ddot{Q}\}=\left[v^{T}\right][\tilde{M}][\ddot{q}\}
$$

However, $\{\ddot{q}\}$ rather than $\left\{\ddot{q}^{\prime}\right\}$ are usually available from the liunch vehicle analysis. Computing $\left\{\ddot{q}^{\prime}\right\}$ as a transient solution of Equation (C-5) is straightforward, but is both costly and unnecessary. For this reason, two approximate alternative solutions are shown next that give very acceptable accuracy at low cost.

1. Approximate Solution 1

Since the right-hard sides of Equations $(C-1)$ and $(C-5)$ are identical, they may be ccmbined to yield the followine exact expression:

$$
\begin{aligned}
{[\tilde{M}]\left\{\begin{array}{c}
\ddot{q}_{c}^{\prime} \\
\ddot{q}_{s}
\end{array}\right\}=} & \left\{\begin{array}{c}
\ddot{q}_{c} \\
\hdashline\left[\omega_{s}^{2}\right]\left[q_{s}^{\prime}\right\}+\left[2 \xi_{s} \omega_{s}\right]\left[\dot{q}_{s}^{\prime}\right\}
\end{array}\right\} \\
& +\left[2 \xi_{c} \omega_{c}\right]\left[\dot{q}_{c}-\dot{q}_{c}^{\prime}\right\}+\left[\omega_{c}^{2}\right]\left\{q_{c}-q_{c}^{\prime}\right\}
\end{aligned}
$$



are identically zero. However, for the elastic modes, these last two terms are small because they express differences between two quantities of global charter having little dependence on the presence of the relatively mall spacecraft. On account of this reasoning, the last two terms or Equation (C-15) can be neglected with little loss in accuracy. This implies:

$$
q_{c} \doteq q_{c}^{\prime} \text { and } \dot{q}_{c} \doteq q_{c}^{\prime}
$$

Also,

$$
q_{s} \doteq q_{s}^{\prime} \text { and } \dot{q}_{s} \doteq q_{s}^{\prime}
$$

Now, Equation $(C-15)$ is approximately written as

$$
[\tilde{M}]\left\{\begin{array}{l}
\ddot{q}_{c}^{\prime} \\
\ddot{q}_{s}^{\prime}
\end{array}\right\} \doteq\left\{\begin{array}{c}
\ddot{q}_{c} \\
{\left[\omega_{s}^{\overline{2}}\right]\left(\bar{q}_{s}^{\prime}\right)+\left[2 \xi_{s} \omega_{s}\right]\left[\dot{q}_{s}^{\prime}\right\}}
\end{array}\right\}
$$

which, when solved with Equation (C-14), yields

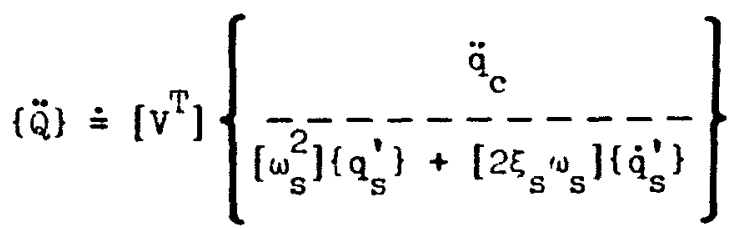

The modul coordinates $\left\{q_{s}^{\prime}\right\}$ and $\left\{\dot{q}_{s}^{\prime}\right\}$ of the elastic simulation of the spacecraft may be obtained from an inexpensive transient solution since they are usually small (of the order of 3 to 6 modes). In this case, the lower partition of Equation. (c-5) can le solved for $\left\{q_{s}^{\prime}\right\}$ and $\left\{\dot{q}_{s}^{\prime}\right\}$ approximately by replacing $\left\{\ddot{q}_{c}\right\}$ by $\left\{\ddot{q}_{c}\right\}$ :

$$
\left\{\ddot{q}_{s}^{\prime}\right\}+\left[2 \xi_{s} \omega_{s}\right]\left\{\dot{q}_{s}^{\prime}\right\}+\left[\omega_{s}^{2}\right]\left\{q_{s}^{\prime}\right\} \doteq-\left[m_{s i}\right]\left[\phi_{i c}\right]\left\{\ddot{q}_{c}(t)\right\}
$$



Bquations (c-22) and (c-23), and making use of $(c-3)$, we get

$$
\left.\left([I]-\Psi_{c R I}\right]\left[M_{11}\right]\left[\phi_{1 C R}\right]\right)\left[\ddot{q}_{c R}^{\prime}\right\} *\left\{\ddot{q}_{c R}\right\}+\left[\phi_{c R I}\right]\left[m_{i s}\right]\left(\ddot{q}_{s}\right\}
$$

$$
+\left[\phi_{c R i}\right]\left[M_{i 1}\right]\left[\phi_{i c E}\right]\left[\ddot{q}_{c E}\right\} \quad(c-24)
$$

In the approximation of Equation $(C-24),\left\{\ddot{q}_{s}\right\}$ is computed in the same manner from Equation (C-19) of the previous approximation. As such, Equations (C-21) and (C-24) along with Equation (C-19) provide an approximate alternative to computing all members of $\{\ddot{q}\}$. Then again, Equation $(C-14)$ is used to compute $\{\ddot{Q}\}$. 


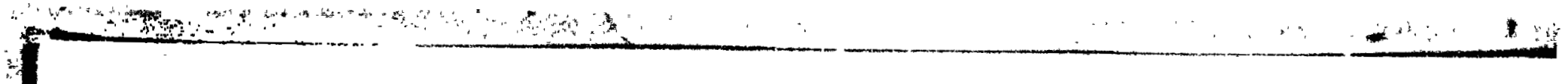

PRECEDIMG PAGE BLANK MOT FILMED

ORIGINAL PAGE IS
OF POOR QUALITIY

APPENDIX D

DISPLACEMENT SPECTRA FOR THE GENERALIZED

RIGID BODY MODES 
In this appendix, an expression is derived for the generalized model displacement spectra, $Q_{g l}$, that should be used for the rigid body modes $(P=0)$ in place of Equation (23) of the main text.

From the lower partition of Equation (13),

$$
\ddot{x}_{2}+2 \xi_{2} \omega_{2} \dot{x}_{2}+(1)_{2}^{2} x=-\ddot{x}_{1}
$$

where, according to Equation (14),

$$
\ddot{x}_{1}=\left(I+M_{s \ell}\right) \ddot{q}_{\ell}
$$

The homogeneous solution of Equation (D-1) having initial conditions $x_{2}(0)=x_{0}$ and $\dot{x}_{2}(0)=\dot{x}_{0}$ is:

$$
x_{2}^{h}(t)=e^{-\xi_{2} \omega_{2} t}\left[\frac{1}{\bar{\omega}_{2}}\left(\dot{x}_{0}+\xi_{2} \omega_{2} x_{0}\right) \sin \bar{\omega}_{2} t+x_{0} \cos \bar{\omega}_{2} t\right]
$$

The particular solution of Equation (D-1) is:

$$
x_{2}^{p}(t)=\frac{1}{\bar{w}_{2}} \int_{\bar{t}=0}^{\bar{t}=t} \ddot{x}_{1}(\bar{t}) e^{-\xi_{2} \omega_{2}(t-\bar{t})} \sin \bar{w}_{2}(t-\bar{t}) d \bar{t}
$$

where

$$
\bar{w}_{2}=\omega_{2} \sqrt{1-\xi_{2}^{2}}
$$

Thus, the total solution of Equation $(D-1)$ is

$$
x_{2}(t)=x_{2}^{h}+x_{2}^{p}
$$

Now, the spacecraft modal response $q_{s}(t)$ to rigid body launch vehicle input $(R=0)$ is given by

$$
q_{s}(t)=\frac{\sqrt{m_{s} l}}{1+M_{s l}} x_{2}(t)
$$


